\title{
The potential of some of the innovative operational procedures for increasing the airport landing capacity
}

\author{
Milan Janic \\ Senior Researcher \\ OTB Research institute \\ Delft University of Technology \\ Jaffalaan 9 \\ 2628 BX Delft \\ The Netherlands \\ Phone: + 31(0) 152787899 \\ Fax: + 31 (0) 152783450 \\ Email:M.Janic@tudelft.nl
}

\begin{abstract}
The ATC (Air Traffic Control) system is established over some designated airspace in order to enable safe, efficient and effective aircraft movements between their origin and destination airports. The main operational performance of these airports has been their airside and landside capacity. In particular, the airport airside capacity includes the capacity of the runway system and surrounding (close) airspace, and the capacities of of taxiways and apron/gate complex. Different concepts of the airport runway system capacity can exist. The first is the concept of the "ultimate" capacity expressed by the maximum number of air transport movements (atms), carried out during a given period of time (usually one hour) under conditions of constant demand for service (atm is either one landing or take-off). The other is the concept of "practical" capacity expressed by the maximum number of atms carried out during a given period of time guaranteeing the average delay per atm within the prescribed limits. Both capacities are calculated respecting the given operational procedures and supportive technologies.

This chapter describes modeling of a potential of some of the innovative operational procedures for safe increasing the "ultimate" runway landing capacity. These are: i) the ATC time-based separation rules applied to landings on a single runway; and ii) the steeper approach procedures applied tp landings on the closely-spaced parallel runways. The modeling implies developing a methodology consisting of the dedicated analytical models for estimating the runway "ultimate" landing capacity under given conditions, which are applied to the selected airports according to the "what-if" traffic scenario approach.
\end{abstract}

Key words: airport, runway landing capacity, innovative operational procedures, the ATC time-based separation rules, the steeper approach procedures, closely-spaced parallel runways. 


\section{Introduction}

Despite continuous efforts by the air transport system operators, regulators, and researchers (academic and consultants), the problem of providing sufficient airport runway capacity to match continuously growing demand safely, efficiently, and effectively has had rather limited success. A[art from growing demand, the specific environmental (mainly noise) constraints at many large airports both in US and Europe have prevented the full utilization of the designed runway capacity. The sharp concentration of atms (air transport movements) (one atm corresponds to one landing or one take off) within the rather short time periods at the hub airports due to operating the hub-and-spoke networks has created sharp peaks causing further already existing imbalance between demand and the available runway capacity. At some other airports one of which is, for example New York La Guardia airport (US), a high demand/capacity imbalance has been created simply because of their attractiveness and not primarily due the type of airline scheduling practice. In addition, specifically in the US, the operation of airports under IMC (Instrument Meteorological Conditions) and VMC (Visual Meteorological Conditions) and the corresponding difference in the ATC (Air Traffic Control) minimum landing distance-based separation rules (IFR - Instrument Flight Rules, and VFR Visual Flight Rules, respectively) have inherently created instability of the airports' declared runway landing capacities and consequently their rather high vulnerability to weather conditions. In Europe, such capacity instability caused by weather has also been relatively high, even though the aircraft landings have been carried out exclusively by applying IFR under both IMC and VMC. As well, the shortage of land for expanding the airport runway capacity at many airports has also contributed to the above-mentioned demand/capacity imbalance there in the long-term. In all cases, this imbalance has created congestion, delays and related airline and air passenger costs.

Under such circumstances, the different ultimately short-term measures for mitigating the demand/capacity imbalance by influencing both demand and capacity have been considered. On the demand side, these have generally been demand management by the slot regulation, auction and trading-off of slots, and eventually congestion charging. On the capacity side in addition to building new runways as the long-term measure, these have mainly included introducing the innovative operational procedures supported by the existing and/or innovative technologies. In general, these latter measures have expected to contribute to reducing the ATC separation minimums between landing aircraft and consequently provide the landing capacity gains within the existing airspace and airport infrastructures (Czerny et al., 2008; Janic, 2008, 2008a; CRS, 2008). The ATC separation minimums have mainly been based on the horizontal distances between landing aircraft, which have been modified respecting the impact of the wake-vortices generated behind the large (heavy) aircraft. The landing aircraft have followed the standardized GS (Glide Slope) angle of $3^{0}$ of the ILS (Instrument Landing System). Such rather inflexible but safe operational pattern has provided the runway landing capacity with the above-mentioned characteristics - insufficient and vulnerable to weather. Consequently, the question is whether some innovative operational procedures supported by the existing and/or new technologies could safely increase the airport runway landing capacity and diminish its vulnerability to weather. Some of these considered are the ATC time-based instead of the current ATC distance-based separation rules between landings on a single runway, and the steeper approach procedures to the closely-spaced parallel runways. Both would be supported by the various ATC (Air Traffic Control) decision-support tools at both tactical and operational level. Specifically, in the US, some of these have included Ground Holding Program (GHP), 
Airspace Flow Program (AFP), Flight Schedule Monitor (FSM), Flight Schedule Analyzer (FSA), and Traffic Management Advisor (TMA) (CRS, 2008).

In addition to this introductory section, this Chapter consists of five other sections. Section 2 describes the above-mentioned innovative operational procedures for increasing and stabilizing the airport runway landing capacity. Section 3 develops a methodology consisting of the dedicated models for estimating the potential contribution of particular innovative procedures to increasing the runway landing capacity. Section 4 presents application of particular models. The final section (5) summarizes some conclusions.

\section{The innovative operational procedures for increasing the runway landing capacity}

\subsection{Background}

The innovative operational procedures for increasing (and stabilizing) the airport runway capacity include the ATC time-based instead of the currently used distance-based separation rules between landings on a single runway and the steeper approach procedures to the closelyspaced parallel runways (Janic, 2008; 2008a).

\subsection{The ATC time-based separation rules for landing aircraft}

\subsubsection{Background}

At oresent, at the US airports, depending on weather, the aircraft landings are carried out either under IMC or VMC. Both types of conditions are specified by two parameters - ceiling and visibility - as shown in Figure 1 (FAA, 2004).

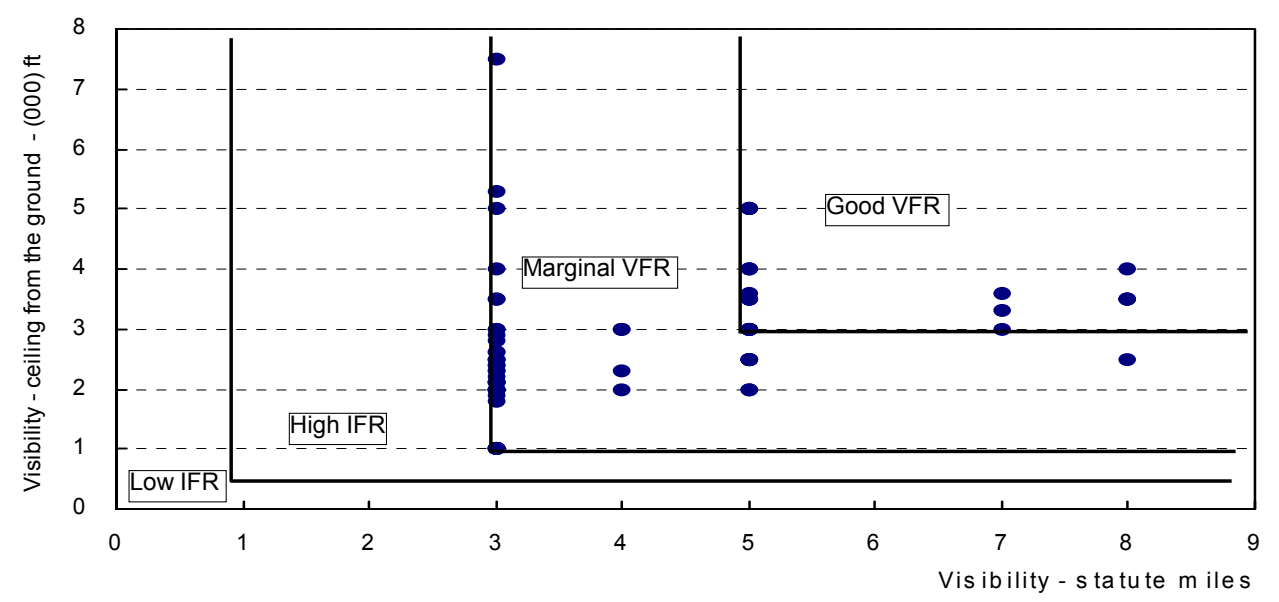

Fig. 1. Characteristics of the meteorological boundary conditions at the 75 selected US airports (Compiled from (FAA, 2004; NASA, 2001)

As can be seen, the critical ceiling is the most diverse when the horizontal visibility is 3 and 5 (statute) miles and relatively homogenous when this visibility is 4, 7 and 8 miles. In addition, most airports operate at the margin between the "high IFR" and the "marginal VFR" (FAA, 
2004; NASA, 2001). Depending on the above-mentioned weather conditions (IMC or VMC), the ATC applies the VFR and IFR corresponding minimum separation rules between landing aircraft given in Table 1 (FAA, 2004).

\begin{tabular}{ccccc}
\hline & \multicolumn{5}{c|}{ IFR } \\
\hline$i / j$ & Small & Large & B757 & Heavy \\
\hline Small & $2.5(3)$ & $2.5(3)$ & $2,5(3)$ & $2.5(3)$ \\
Large & 4.0 & $2.5(3)$ & $2.5(3)$ & $2,5(3)$ \\
B757 & 5.0 & 4.0 & 4.0 & 4.0 \\
Heavy & 6.0 & 5.0 & 5.0 & 4.0 \\
\hline Small & LFR & & Heavy \\
\hline Small & 1.9 & 1.9 & B757 & 1.9 \\
Large & 2.7 & 1.9 & 1.9 & 1.9 \\
B757 & 3.5 & 3.0 & 3.0 & 2.7 \\
Heavy & 4.5 & 3.6 & 3.6 & 2.7 \\
\hline
\end{tabular}

Table 1. The FAA (ICAO) minimum separation rules between landing aircraft (nm) Compiled from: (FAA, 2004; NASA, 1999, 2001)

As can be seen, the current IFR separations applied under IMC are for about 40 per cent stricter than the VFR separations applied under VMC. Both separation rules generally eliminate the impact of the wake vortices of the leading aircraft on the trailing aircraft in particular combinations of landing sequences on the same runway. Under an assumption that the potential exposure of the trailing aircraft to the wakes generated by the leading aircraft in a given landing sequence is nearly the same for both types of separations, the question is: "Why is such a distinction between the VFR and the IFR separations?". The possible answer could be that under VMC, the trailing aircraft fly on the principle "see and be seen" by keeping just a sufficient distance to avoid the wake vortex hazard from the leading aircraft. Under IMC, in addition to the basic separation rules required to avoid the wake vortices, the ATC introduces the additional "buffers" to compensate the cumulative system error in estimating the aircraft position(s). These positions are visualized for the ATC controllers thanks to the sophisticated radar systems. The influence of the two categories of separation rules on the landing capacities, i.e arrival rates, at the selected US airports are shown in Figure 2. 


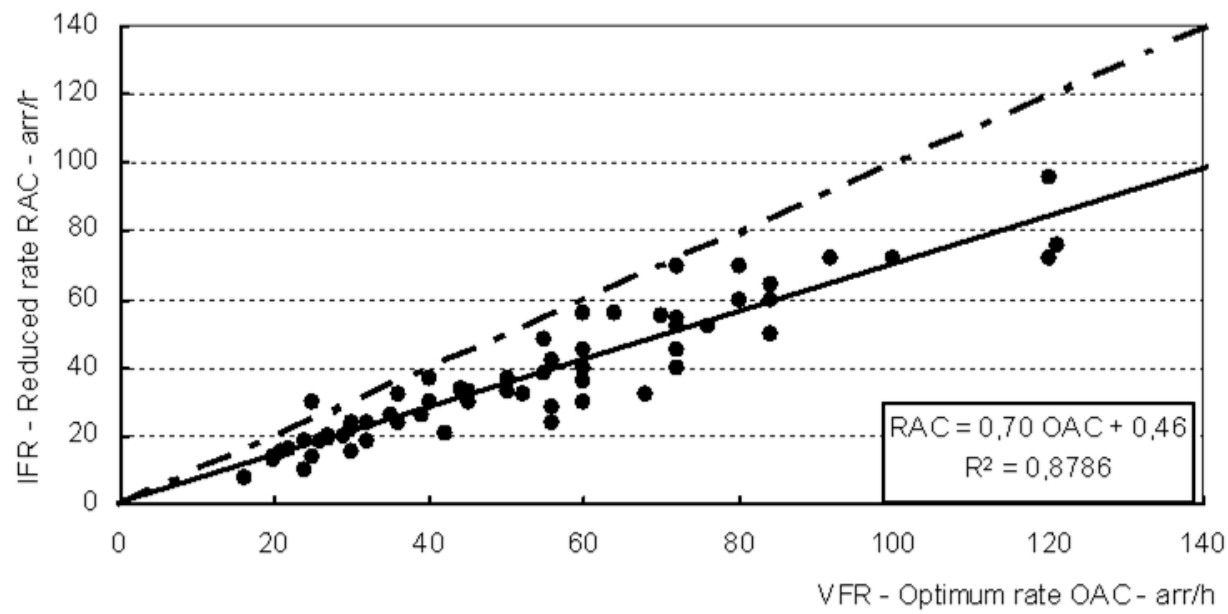

Fig. 2. Relationship between the IFR and VFR landing capacity at the selected US airports (Compiled from: FAA, 2004; NASA, 2001)

As can be seen, a rather strong linear relationship between the IFR and VFR landing capacities (arrival rates), with an average difference of about 30 per cent exists, i.e. the IFR landing capacities generally amount up to about 70 per cent of the corresponding VFR landing capacities. In Europe, independently of the weather conditions, the landings are carried out exclusively according to the IFR separations in Table 1 (EEC, 2005). Consequently, the question is if it is possible to set up the time-based separation rules, which would be standardised respecting the true (dynamic) behaviour of the wake vortices under all weather conditions. In general, these separation rules are expected to provide the shorter minimum time-distance intervals between successive landing aircraft and consequently increase the current distancedependent runway landing capacity while maintaining it rather stable subject to weather changes. This could be possible if more precise monitoring of the true behaviour of the wake vortices behind particular aircraft would be enabled to pilots, ATC, and/or both.

\subsubsection{The "wake reference airspace"}

Monitoring the true behaviour of the wake vortices, i.e. dynamically, requires defining the "wake reference airspace" used for the final approach and landing on a given runway. In general, this space consists of two parts: i) the "wake vortex corridor", i.e. the airspace of shape of a horizontal prism, which spreads along the extended centreline of the runway; and ii) the SHA (Simplified Hazard Area) in which the wake vorteuces generated by a given aircraft remains until they decay and/or vacate the "wake reference airspace" (Janic, 2008; ONERA/DOTA, 2005). The "wake vortex corridor" begins at FAG (Final Approach Gate), which is usually defined as the waypoint or by the radio-navigational aid (VOR/DME). Ur ends at the runway touchdown area. Figure 3 shows the simplified three-dimensional scheme of the "wake reference airspace". 


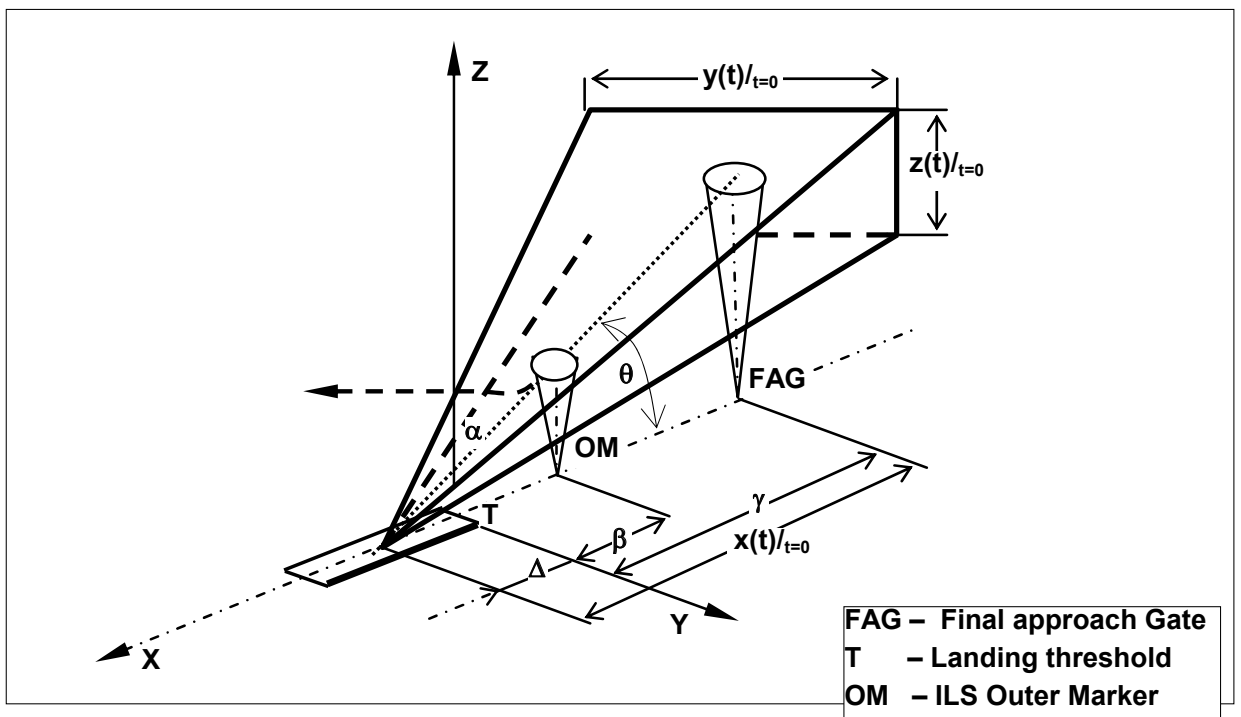

Fig. 3. Three-dimensional scheme of the "wake reference airspace" (Compiled from (Janic, 2008)

where $\gamma$ is the length of the "wake vortex corridor"; $\Delta$ is the horizontal distance between the FAG (Final Approach Gate) at the beginning of the "wake vortex corridor", and the runway landing threshold T; OM and MM are Outer and Middle marker, respectively, of ILS (Instrument Landing System); $x(t), y(t)$, and $z(t)$ are longitudinal, horizontal, and vertical coordinates, respectively, of the "wake reference airspace", depending on time $(t) ; \beta$ is the horizontal distance between the location of the OM and the runway-landing threshold $T \alpha$ is the angle between the axis of the "wake vortex airspace" and one of its sides in the horizontal plane; and $\theta$ is the nominal angle (ILS Glide Slope) of the aircraft approach path in the "wake vortex airspace".

As mentioned above, ILS provides the approaching and landing aircraft with primary navigation. In the future, the Cockpit Display Traffic Information (CDTI) system on-board the aircraft supported by the ADS-B device will be used for easier self-managing the arrival procedure individually and relative to other close traffic. The ATC usually uses the highly sophisticated radar system for monitoring the arriving traffic. For example, the Precision Radar Monitoring (PRM) system is one of them. In addition, monitoring and prediction of the wake vortex behavior in the "wake reference airspace" is and will be carried out by the current and forthcoming technologies and systems both on the ground and on board the aircraft (Choroba, 2003; Wilkenmans and Desenfans, 2006). The most well-known current system on the ground is Aircraft Vortex Spacing System (AVOSS) currently operating at Dallas Fort Worth airport (US). The system provides the dynamic spacing criteria between aircraft approaching the single runway along a pre-defined corridor based on the prediction of the wake vortex position and strength dependent on the current weather conditions. The wake attribute, which first clears the corridor at a certain ("reference") profile, defines the distance separation criterion for a given aircraft. The standardization and operationalization of such ATC distance-based into the ATC time-based separation rules will likely require the full development of the active (dynamic) forthcoming wake vortex advisory systems such as ATC WAKE, WAKEVAS, and WVWS The particular components of these systems both onboard the aircraft and at the ATC working desk 
will enable monitoring and predicting the wake vortex behavior within the entire "wake reference airspace" and exchanging the information between pilots and controllers online, i.e. automatically via data link. The information on the wake vortex of the preceding aircraft would be presented to the crew either on the Navigational or Primary Flight Display containing the wake's strength and prospective behavior (movement) within the "wake reference airspace". Under such circumstances enabling pilots to monitor the wake vortex of the aircraft they follow on the cockpit screen instead of looking at the aircraft itself, which they cannot see under IMC, the separation between the landing aircraft could become purely the dynamic time-based separation, and, in terms of the distances, closer to the today's VFR minimum distance-based separation intervals mainly applied to the US airports (Choroba, 2003; Wilkenmans and Desenfans, 2006).

\subsection{The steeper approach procedures}

\subsubsection{Background}

Different configurations of parallel runways are used at busy European and US airports. In Europe, the four busiest continental hubs operate parallel runways: Frankfurt-Main (Germany) a pair of closely-spaced (dependent), and London Heathrow (UK), Paris Charles de Gaulle (France), and Amsterdam Schiphol airport (The Netherlands) a pair, two pairs, and three pairs of the far-spaced (independent) parallel runways, respectively. Currently, at the U.S. the busiest hub airports operate 28 pairs of closely-spaced, 10 pairs of the intermediate-s[aced, and 28 pairs of the far-spaced parallel runways (NASA, 1998). In addition to the above-mentioned characteristics valid for a single runway, in case of parallel runways, the wakes can move from the "wake reference airspace" of one runway to this airspace of the adjacent runway(s) at the speed almost proportional to the speed of crosswind. If the wakes do not sufficiently decay before reaching the adjacent runway, they can create a hazard for the aircraft there, thus making operations on both runways dependent on each other (Burnham, 2002; FAA, 2004; Hammer, 2000; NASA, 2001). Under such circumstances, under VMC, the ATC applies the VFR to the approaches to the parallel runways spaced by $2500 \mathrm{ft}(762 \mathrm{~m})$ and less by assuming that the wakes generated along the "wake reference airspace" of one runway will never reach this airspace of the adjacent (parallel) runway. This makes the two runways operationally independent on each other (FAA, 2004; Janic, 2008a; LMI, 2004). Under IMC, the ATC exclusively applies the IFR horizontal separation rules in Table 1 between the aircraft approaching to either of the closely-spaced parallel runways, thus making both runways to operate as dependent of each other, i.e. as a single runway. In such case, the CNAP (Conventional Approach Procedure) is performed (Janic, 2008a).

\subsubsection{The characteristics of Steeper Approach Procedure (SEAP)}

In order to mitigate the above-mentioned dependency of closely-spaced parallel runways the procedures for pairing the arriving aircraft under IMC similarly as under VMC have been considered in both Europe and US. In Europe this has been Staggered Approach Procedure (SGAP) with displaced landing threshold at one of the closely-spaced parallel runways at Frankfurt Mian airport (Germany) (Fraport, 2004). In the US these have been: Simultaneous Offset Instrument Approach/Precision Runway Monitoring (SOIA/PRM), and the most advanced but still under the conceptual development NASA/FAA TACEC (Terminal Area Capacity Enhancing Concept) (Burnham, 2002; Cotton et al., 2001; EEC, 2005), These and an additional innovative procedure called Steeper Approach Procedure (SEAP) use both ATC 
horizontal and vertical separation rules simultaneously. Figure $4(\mathrm{a}, \mathrm{b})$ shows the principal difference between CNAP and SEAP performed under IMC (Janic, 2008a).

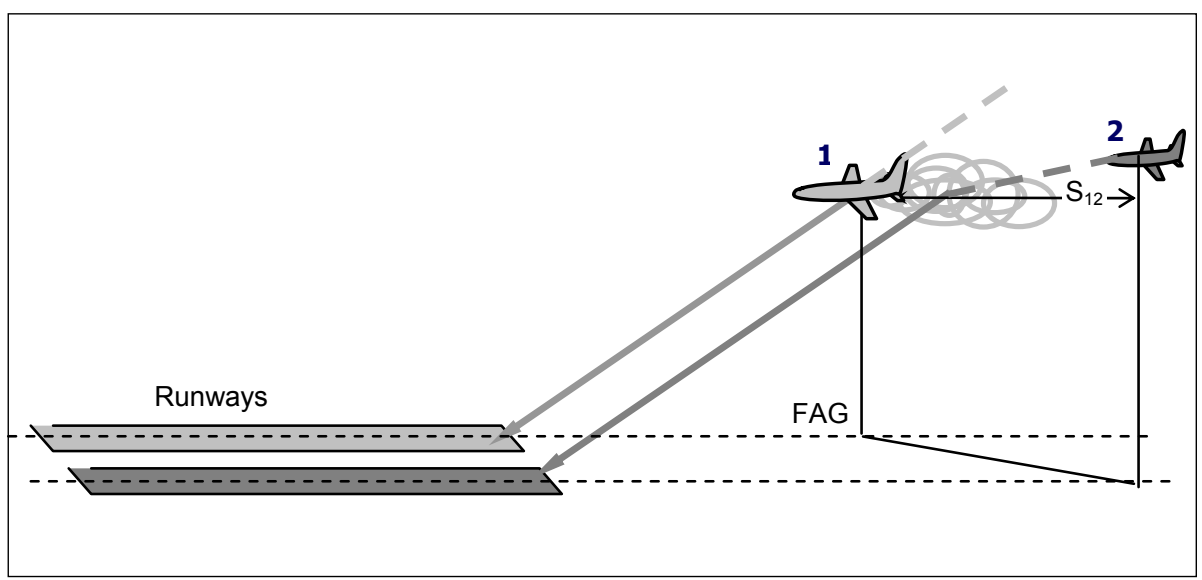

a) Conventional Approach Procedure (CNAP)

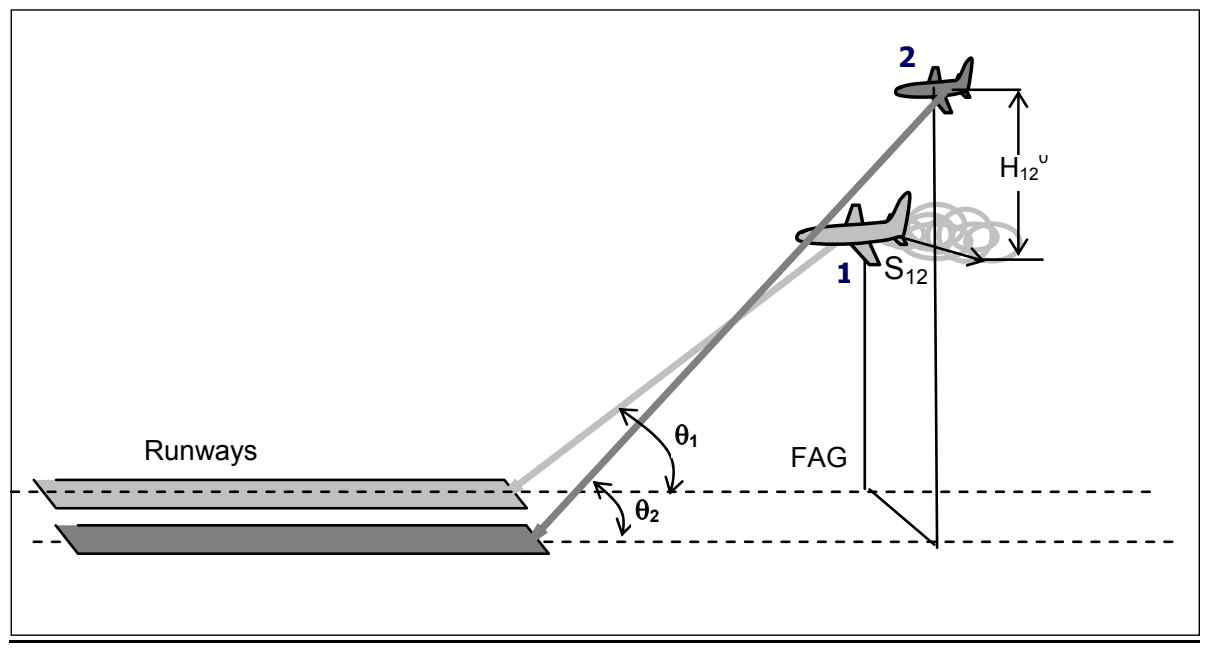

b) Steeper Approach Procedure (SEAP)

Fig. 4. The CNAP and SEAP to the closely spaced parallel runways under IMC (Compiled from: Janic, 2008a)

The Steeper Approach Procedure (SEAP) could be considered as the prospective approach procedure under IMC in cases when it is necessary to avoid obstacles in the final approach airspace, if it is not possible to displace the landing threshold of one of two (closely spaced) parallel runways, but if it is needed to eventually relatively substantively increase the runway system capacity. Currently, the SEAP is applied to some single runway regional airports (six in Europe) mainly for avoiding obstacles and/or eventually reducing the noise burden (EEC, 2005). This procedure has never been considered for application to the closely-spaced parallel 
runways for any of the above-mentioned purposes and particularly not for eventual increasing of the runway system capacity under IMC. Even the above-mentioned future concept (TACEC) does not consider the final approaches at different (and steeper) GS angles than nowadays. Consequently, at this preliminary stage the SEAP is characterized as follows:

\section{- Technologies}

The SEAP applied to the closely-spaced parallel runways can be based on two pairs of ILSs (or GNSS supporting ADS-B in the future) each attached to one of the runways or a pair of MLSs each serving a single runway. For a given runway, one ILS provides the standard GS angle of $3^{0}$ and the other the steeper GS angle of $5-7^{0}$. A single MLS provides simultaneously both GS angles within a given range: $3-7^{0}$ (This will also be possible when GNSS and ADS-B will be available) (Rossow, 2003;TC, 2004). The ILSs are preferably of the category IIIb or IIIc (i.e. with zero Decision Height (DH) and Runway Visual Range- (RVR) of 50ft or 0ft, respectively) thus enabling also the auto-landing under the worst visibility conditions (ICAO, 1996). Each ILS has different LLZ (Localizer) frequency coupled with the GP (Glide Path) frequency, which prevents interference between the ILSs serving the same runway. Thus, given aircraft can perform either the standard or the steeper approach and landing procedure independently, by using existing ILS avionics and Flight Management (FM) auto-landing system. MLS also enables the similar auto landing opportunities. In addition, the ATC can also use PRM for monitoring the arriving traffic. As well, other technologies improving the situational awareness both at aircraft and on the ground could be gradually implemented (EEC, 2005; ICAO, 1996; Janic, 2006, 2008a). Ub addition, the lighting system on each parallel runway must be appropriately calibrated respecting the different ILS or MLS GS angles. This might appear unfeasible causing the pilot confusion, and thus being considered as insufficiently safe. The auto-landing could mitigate or even eliminate this concern.

\section{- Operations}

The SEAP implies that the arriving aircraft can use either the standard or the steeper ILS GS angle while approaching to the closely-spaced parallel runways under IMC. In particular, if the aircraft pairing is made similarly as under VMC or the SOIA/PRM, the leading aircraft can be assigned the standard and the trailing aircraft the steeper GS angle. Figure $4 \mathrm{~b}$ shows the simplified typical scheme when at the moment of pairing the heavy - leading (1) and small trailing (2) aircraft approaching to the closely-spaced parallel runways. As can be seen, the leading aircraft (1) approaches to the right parallel runway at the standard GS angle $\theta_{1}$; the trailing aircraft (2) approaches to the left runway at the steeper GS angle $\theta_{2}\left(\theta_{1}<\theta_{2}\right)$. The leading aircraft (1) and trailing aircraft (2) are appropriately vertically separated by the ATC vertical separation rules $\mathrm{H}_{12}{ }^{0}$ at the moment of pairing at FAG (Final Approach Gate) of aircraft (1). This initial vertical separation does not exclude some horizontal separation $S_{12}$, which might be unnecessary. In addition, when the condition regarding the aircraft speeds and GS angles is fulfilled (i.e. $v_{1}>v_{2} \sin \theta_{2} / \sin \theta_{1}$ ), the initial minimum vertical separation $H_{12}{ }^{0}$ continuously increases - until the leading aircraft (1) lands. Under such circumstances the aircraft (2) will always stay above the aircraft (1), thus completely avoiding the hazard of its wakes staying all the time below its final approach trajectory. Nevertheless, the hazard from wakes along the same approach path still requires application of the ATC longitudinal (in-trail) separation rules. 
- Traffic complexity

Performing the SEAP and CNAP simultaneously on the same runway(s) may increase the traffic complexity and consequently the workload of ATC controllers and pilots. However, the updated decision supporting tools such as CTAS (Centre/TRACON Automation System) and Integrated Arrival/Departure manager on the one hand, and CDTI on the other, may compensate such increased workload, respectively (Janic, 2006).

\section{- Standardization}

The SEAP is not the standardized ICAO procedure such as CNAP. Therefore, it needs approval from the local airport and national aviation.

- The aircraft certification

Specifically, the aircraft should be technically capable and consequently certified for SEAP similarly as they are currently certificated for CEAP. The certification could include only discrete but also the continuous GS angles within a given range. Since most Boeing and Airbus aircraft do not have such certification, the related costs of additional certification might be relatively high, of course if the aircraft are considered capable for being certified for the SEAP safely ${ }^{1}$. While following the steeper approach trajectory, the aircraft need higher descent speed, which in turn reduces the horizontal component of the resultant speed and thus seemingly increases the wake-vortex. In order to prevent the impact the resultant approach speed should be increased to compensate the higher vertical component authorities.

\section{- Training the staff}

The pilots and ATC controllers must be appropriately trained. One aspect of training of pilots is preparation of the aircraft full landing configuration in the SEAP earlier than in the CNAP, which includes intercepting the steeper GS angle, and stabilizing and keeping the constant approach speed at the lower thrust. Training of the ATC controllers implies familiarizing with application of different combinations of separation rules and eventually with a potential for handling an increased number of missed approaches.

\section{- Passenger comfort}

The vertical speed during CNAP of about $500-800 \mathrm{ft} / \mathrm{min}$ currently appears comfortable for passengers. Under the same circumstances, increase in the vertical speed during SEAP for about $80 \%$ as compared to CNAP might be uncomfortable.

\section{A methodology for estimating the potential of innovative procedures to increasing the runway landing capacity}

\subsection{Background}

The methodology for estimating the potential contribution of the above-mentioned innovative procedures to the airport runway landing capacity consists of two dedicated models: i) the model when the ATC time-based separation rules are applied; and ii) the model when the SEAP

\footnotetext{
1 The earliest, De Havilland DHC- 6 and DHC- 8 had been certified as the STOL (Short Take Off and Landing) aircraft. Later, the regional aircraft Cessna Citation, BAe RJ 85/100, Fokker 50, Dornier 328, Embraer ERJ 135/170, and recently the larger Airbus A318/319 have been certified for the SEAP (EEC, 2005; TC, 2004).
} 
in addition to CNAP is applied to the closely-spaced parallel runways. The models have the analytical structure enabling carrying out the sensitivity analysis with respect to changes of the most influencing factors.

\subsection{Previous research}

Modelling of the airport ultimate (runway) capacity has occupied the airport, ATC, and airline operators, planners, analysts and academics for a long time. These efforts have resulted in developing the numerous analytical and simulation models, which could be classified into two broad classes for: i) calculating the (runway) capacity of individual airports and of the airport network(s) (Odoni and Bowman, 1997); and ii) optimization of utilization of the airport (runway) available capacity under changing influencing factors and conditions (Andreatta and Romanin-Jacur, 1987; Bianco and Bielli, 1993; Richetta and Odoni, 1993, 1994; Richetta, 1995; Terrab and Odoni, 1993; Vranas et al., 1994).

Specifically, the analytical models for calculation of the airport runway capacity have provided the two-value parameter - one for the arrival and another for the departure capacity (Blumstein, 1959; Donahue, 1999; Gilbo, 1993; Harris, 1972; Hockaday and Kanafani, 1974; Janic and Tosic, 1982; Janic, 2006; Newell, 1979; Swedish, 1981). Some other models such as the FAA Airport Capacity Model, LMI Runway Capacity Model, and DELAYS as 'Quasi-Analytical Models of Airport Capacity and Delay', developed mainly for the airport (runway) planning purposes and based on the analytical single-runway capacity model, have calculated the socalled "capacity coverage curve" including the associated aircraft delays (Gilbo, 1993; Newell, 1979). In parallel, separate models of the ultimate capacity of the airport apron/gate complex and the system of taxiways have been developed. Recently, these analytical models have been integrated into the 'airport integrated-strategic planning tool' (EEC, 2005). An additional integration has however been achieved by developing the computer-supported simulation models for calculating the airport capacity and delay at i) Low (HERMES and The Airport Machine), ii) Intermediate (NASPAC, FLOWSIM and TMAC), and iii) High Level of Detail (TAAM and SIMMOD) (Ignaccolo, 1993; Janic, 2001; Odoni and Bowman, 1997; Swedish, 1981; $\mathrm{Wu}$ and Caves, 2002). In comparison to the analytical models, these models have studied the airport airside operations in much greater details. In some cases, they have seemed to require relatively long time for familiarization, time-consuming preparation of input, consequently relatively high cost, and produced too detailed output, which paradoxically made the strategic planning choices more complex and time consuming than otherwise (Odoni and Bowman, 1997; Stamatopoulos et al., 2004). However, the efforts on further refining existing and developing new models offering estimation of the potential of some of the innovative operational procedures and technologies for increasing the airport runway landing capacity have been made. They have resulted in developing the analytical models for estimating the "ultimate" landing capacity for the cases elaborated in this Chapter, i.e. the ATC time-based separation rules and and the steeper approach procedures, both considered as elements of the current NextGen (US) and SESAR (Europe) programmes (http://vams.arc.nasa.gov/activities/ tacec.html). (Janic, 2006, 2008, 2008a).

\subsection{Objectives and assumptions}

The objectives of the research described in this Chapter are to develop the methodology consisted of the dedicated analytical models, which will enable estimating the potential of the selected innovative operational procedures and technologies to increase the airport runway 
landing capacity under given conditions. In addition, each model should enable carrying out the sensitivity analysis of the capacity with respect to changes of the most important influencing factors. Consequently, the methodology is based on the following assumptions (Janic, 2006, 2008; 2008a, 2009):

- The runway system consisting of a single and/or a pair of the closely-spaced parallel runways with the specified geometry used exclusively for landings is considered;

- The aircraft arrive at the specified locations of their prescribed arrival paths almost precisely when the ATC (controller) expects them, i.e. the system is considered as "the error free";

- The occurrence of particular aircraft categories in particular parts are mutually independent events;

- The arrival mix characterized by the weight (i.e. the wake-vortex category) and approach speed of particular aircraft categories is given;

- The aircraft approach speeds along particular segments of the "wake reference airspace" are constant.

- The influence of the weather conditions on the wake vortex behavior for a given landing sequence is constant during the aircraft staying in the "wake reference airspace";

- The ATC uses the radar-based longitudinal and horizontal-diagonal, and vertical separation rules between the arriving aircraft;

- Assignment of CNAP/SEAP depends on type of the arrival sequence(s) in terms of the aircraft wake-vortex category, approach speed, and capability to perform SEAP in the latter case;

- The successive arrival aircraft approaching to the closely-spaced parallel runways, are paired and alternated on each runway; and

- Monitoring of the current, and prediction of the prospective behavior of the wake vortices in the "wake reference airspace" is reliable thanks to the advanced technologies;

\subsection{Basic structure of the models}

The models developed possess a common basic structure, which implies determining the "ultimate" landing capacity of a given runway(s) as the reciprocal of the minimum average "inter-arrival" time of passing of all combinations of pairs of landing aircraft through a given "reference location" selected for their counting during a given period of time (Bluemstein, 1959). In the given context, the minimum average inter-arrival time enables maximization of the number of passes through the "reference location", which is usually the runway landing threshold. The period of time is $1 / 4,1 / 2$, and/or most usually 1 hour.

Consequently, the basic structure of the model using the ATC time-based instead of the ATC distance-based separation rules between landing aircraft on a single runway is based on the traditional analytical model for calculating the "ultimate" runway landing capacity as follows(Blumstein, 1959; Janic, 2001):

$$
\lambda_{a}=T / \sum_{i j} p_{i a} t_{i j \min } p_{j}
$$


where
${ }_{a} t_{i j m i n}$
is the minimum inter-arrival time of the aircraft pair (i) and ( $j$ ) at the runway landing
$p_{i}, p_{j} \quad$ is the proportion of aircraft types $(i)$ and $(j)$ in the landing mix, respectively;
$T$ are the periods of time (usually one hour).

In the case of the SEAP on the closely-spaced parallel runways, let's assume yajy yjere are two aircraft landing sequences: i) the aircraft sequence (ij) is to land on RWY1; and ii) the aircraft sequence $(\mathrm{kl})$ is to land on RWY2. Since the occurrences of particular aircraft categories are mutually independent events on both runways, the probability of occurrence of the "strings" of aircraft $(i k j)$ and $(k j l)$ can be determined as follows (Janic, 2006, 2008a):

where

$$
p_{i j / k}=p_{i} p_{k} p_{j} \text { and } p_{k l / j}=p_{k} p_{j} p_{l}
$$

$p_{i}, p_{k}, p_{j}, p_{l} \quad$ is the proportion of aircraft categories $(i),(k),(j)$ and $(l)$ in the mix, respectively.

Given the minimum inter-arrival time at the landing threshold of RWY1 and RWY2 as a $t_{i j / k / m i n}$ and ${ }_{a} t_{k l j / m i n}$, respectively, and the probabilities $p_{i j / k}$ and $p_{k l / j}$ for all combinations of the aircraft sequences $(i k j)$ and $(k j l)$, respectively, the average inter-arrival time at the threshold of RWY1 and RWY2 in Figure $4 \mathrm{~b}$ as the "capacity calculating locations" can be computed as follows (Janic, 2006, 2008s):

$$
\bar{t}_{a 1}=\sum_{i k j k}{ }_{a} t_{i j / k / \min } p_{i j / k} \text { and } \bar{t}_{a 2}=\sum_{k j l}{ }_{a} t_{k l / j / \min } p_{k l / j}
$$

Then, the "ultimate" arrival capacity of a given pair of the closely-spaced parallel runways can be calculated separately for each runway as (Janic, 2006):

$$
\lambda_{a 1}=T / \bar{t}_{a 1} \text { and } \lambda_{a 2}=T / \bar{t}_{a 2}
$$

The total landing capacity for the runway system can be calculated as the sum of the individual capacities of each runway.

\subsection{Determining the minimum interarrival time(s) at the "reference location"}

\subsubsection{The ATC time-based separation rules}

The minimum time-based separation rules for the aircraft landing on a single runway are determined by modeling the wake-vortex behavior in the "wake reference airspace", setting up the dynamic time-based separation rules, and calculating the inter-arrival times of particular sequences of landing aircraft at the "reference location", i.e. the runway landing threshold $T$ in Figure 3 (Janic, 2008). 


\subsubsection{The wake vortex behavior}

The wake vortex appears as soon as the lift on the aircraft wings is created. The investigations so far have shown that the wakes behind the aircraft decay over time generally at more than proportional rate, while simultaneously descending below the aircraft trajectory at a certain descent speed. Without crosswind they also move from the aircraft trajectory at a self-induced speed of about $5 \mathrm{kt}$ (knots). Otherwise, they move according to the direction and speed of the crosswind (Shortle and Jeddi, 2007).

Modeling the wake-vortex behavior includes determining its strength, i.e. the root circulation, the "reference time", decaying pattern, decent speed, and the movement influenced by the ambient weather.

The wake strength - the root circulation at time $(\mathrm{t})$. This can be estainated as follows:

$$
\Gamma_{0}(t)=\frac{4 M g}{\rho v(t) B \pi}
$$

The wake reference time, i.e. the time for the wake to descend for one wing span at time (t). This can be estimated as follows:

$$
t^{*}(t)=\frac{\pi^{3} B^{2}}{8 \Gamma_{0}(t)}=\frac{\rho \pi^{4} B^{3} v(t)}{32 M g}
$$

The wake-decaying pattern. This is estimated as follows:

$$
\Gamma(t)=\Gamma_{0}(t)\left(1-\frac{t}{k t^{*}(t)}\right)
$$

If the safe wake strength is $\Gamma^{*}$, the time the wake needs to decay to this level, $\tau_{d}\left(\Gamma^{*}\right)$ can be determined from expression (5c) as follows:

$$
\tau_{d}\left(t, \Gamma^{*}\right)=k t^{*}(t)\left(1-\frac{\Gamma^{*}(t)}{\Gamma_{0}(t)}\right)
$$

The wake's self-induced descent speed. This is determined as follows:

$$
w(t)=\frac{2 \Gamma(t)}{\pi^{2} B}=\frac{2 \Gamma_{0}(t)\left[1-t / k t^{*}(t)\right]}{\pi^{2} B}
$$


where

$M \quad$ is the aircraft (landing) mass (kg);

$g \quad$ is the gravitational acceleration $\left(\mathrm{m} / \mathrm{s}^{\mathrm{\alpha}}\right)$;

$\rho \quad$ is the air density near the ground $\left(\mathrm{kg} / \mathrm{m}^{5}\right)$;

$v(t) \quad$ is the aircraft speed at time $(t)(\mathrm{m} / \mathrm{s})$;

$B \quad$ is the aircraft wingspan (m); and

$k \quad$ is the number of the reference time periods after the wakes decay to the level of the natural turbulence near the ground $\left(70 \mathrm{~m}^{2} / \mathrm{s}\right)(k=8-9)$.

\section{The impact of ambient weather}

The ambient weather is characterized by the ambient wind, which can influence the wake vortex behaviour in the "wake reference airspace". This wind is characterized by the crosswind and headwind components as follows.

- $\quad$ Crosswind:

The crosswind can be determined as follows:

$$
V_{c w}(t)=V_{w}(t) \sin \left(\varphi_{w}-\varphi_{a}\right)
$$

The wake vacates the "reference profile" at almost the same speed as the crosswind.

\section{- $\quad$ Headwind:}

The headwind can be determined as follows:

where

$$
V_{h w}(t)=V_{w}(t) \cos \left(\varphi_{w}-\varphi_{a}\right)
$$

$V_{w}(t) \quad$ is the wind reported by the ATC at time $(t)$;

$\varphi_{w} \quad$ is the course of the wind $\left({ }^{v}\right)$;

$\varphi_{a} \quad$ is the course of the aircraft $\left(^{(}\right)$.

The headwind does not directly influence the wake descent speed (rate) but does move the wake from the ILS GS and thus increases its vertical distance from the path of the trailing aircraft. This vertical distance increases linearly over time and in proportion to the headwind as follows:

$$
\Delta z_{h w}(t)=V_{h w}(t) * t * \operatorname{tg} \theta
$$

where all symbols are as in the previous expressions. 


\subsubsection{The dynamic time-based separation rules}

Let $\tau_{i j / \min }(t)$ be the minimum time-based separation rules between the leading aircraft (i) and aircraft $(j)$ in the landing sequence (ij) at time $(t)$. Currently, this time depends on the ATC distance-based separation rules (either IFR or VFR) implicitly including the characteristics of the wake vortex behavior, and the aircraft approach speeds (see Table 1). The main idea is to make these time separations explicitly based on the current and predicted characteristics and behavior of the wake vortex generated by the leading aircraft $(i)$ in the given sequence (ij). The characteristics and behavior of the wake vortex include its initial strength and time of decay to a reasonable (i.e. safe) level, and/or the time of clearing the given profile of the "wake reference airspace" either by the self-induced descend speed, headwind, self-induced lateral speed, and/or crosswind.

Let $\tau_{i j}(t), \tau_{i y}(t)$ and $\tau_{i z}(t)$, respectively, be the time separation intervals between the aircraft $(i)$ and (j) based on the current ATC distance-based separation rules in Table 1, and the predicted times of moving the wakes of the leading aircraft (i) either horizontally or vertically at time ( $t$ ), out of

the "wake reference airspace" at a given location. In addition, let $\tau_{i d / j}(t)$ be the predicted time of decay of the wake of the leading aircraft $(i)$ to the level acceptable for the trailing aircraft $(j)$ at time $(t)$. Refering to Figure 3, these times can be estimated as follows:

$$
\begin{aligned}
& \tau_{i j}(t)=\delta_{i j}(t) / v(t) \\
& \tau_{i y}(t)=Y_{i}(t) / V_{c w}(t) \\
& \tau_{i z}(t)=\min \left[Z_{i}(t) / w_{i}(t) ; \Delta z_{i j / \min }(t) / V_{h w}(t) \operatorname{tg} \theta\right] \\
& \tau_{i d / j}\left(t, \Gamma^{*}\right)=k t_{i}^{*}(t)\left[1-\Gamma_{j}^{*} / \Gamma_{0 i}(t)\right]
\end{aligned}
$$

where

$\delta_{i j}(t) \quad$ is the minimum ATC distance-based separation rules applied to the landing sequence $(i j)$ at time $(t)$;

$v_{j}(t) \quad$ is the average approach speed of the trailing aircraft $(j)$ at time $(t)$; and

$\Delta z_{i j / \min }(t) \quad$ is the minimum vertical separation rule between the aircraft $(i)$ and $(j)$ at time $(t)$.

Other symbols are analogous to those in the previous expressions. Expression (6a) indicates that the time the wakes of the leading aircraft (i) take to move out of the given "reference profile" does not depend on the type of trailing aircraft $(j)$. However, the decaying time of the wakes from the leading aircraft (i) depends on its strength, which has to be acceptable (i.e. safe) for the trailing aircraft (i). Consequently, at time $(t)$, the trailing aircraft $(j)$ can be separated from the leading aircraft $(i)$ by the minimum time separation rules as follows:

$$
\tau_{i j / \min }(t)=\min \left[\tau_{i j}(t) ; \tau_{i y}(t) ; \tau_{i z}(t) ; \tau_{i d / j}\left(t, \Gamma^{*}\right)\right]
$$

- If $v_{i} \leq v_{j}$, the minimum time separation rule $\tau_{i j / m i n}(t)$ should be established when the leading aircraft (i) is at the runway landing threshold $T$ in Figure 3, i.e. at time $t=\gamma / v_{i}$. In 
addition, the following condition must be fulfilled: $\tau_{i j / m i n}(t) \geq t_{a i}$, where $t_{a i}$ is the runway occupancy time of the leading aircraft (i).

- If $v_{i}>v_{j}$, the minimum time separation rule $\tau_{i j / \min }(t)$ should be established when the leading aircraft (i) is just at FAG (Final Approach Gate) in Figure 3, i.e. at time $t=0$. This is based on the fact that the faster leading aircraft (i) will continuously increase the distance from the slower trailing aircraft $(j)$ during the time of approaching the runway.

\subsubsection{The minimum inter-arrival times between landings}

The minimum inter-arrival times for the aircraft sequences (i) and $(j)$ at the landing threshold can be determined based on expression (6b) as follows:

$$
{ }_{a} t_{i j / \min }=\left\{\begin{array}{ll}
\tau_{i j / \min }(t=0)+\gamma\left(1 / v_{j}-1 / v_{i}\right) & \text { for } v_{i}>v_{j} \\
\max \left[t_{a i} ; \tau_{i j / \min }\left(t=\gamma / v_{i}\right)\right] & \text { for } v_{i} \leq v_{j}
\end{array}\right\}
$$

where $\tau_{i j / \min }(t)$ is determined according to expression $6(\mathrm{a}, \mathrm{b})$.

At time $t=0$, when the leading aircraft (i) is at FAG, the "wake reference profile" is as its greatest, which implies that the wakes need the longest time to vacate it by any means. At time $t$ $=\gamma_{i} v_{i}$, when the leading aircraft $(i)$ is at the landing threshold, the "wake reference profile" is the smallest, which implies that the wakes need much shorter time to vacate it (see Figure 3).

\subsubsection{The Steeper Approach Procedure (SEAP)}

The minimum inter-arrival times between the aircraft landing on the closely-spaced parallel runways are estimated respecting the fact that they can perform both CNAP (Conventional Approach Procedures) and SEAP (Steeper Approach Procedures). At both, the ATC applies the longitudinal (i.e., in-trail) separation rules to the aircraft on the same and the horizontaldiagonal and/or the vertical separation rules to the aircraft on the different (parallel) approach trajectories.

\subsubsection{Scenario for performing SEAP}

Simultaneous performing of CNAP and SEAP at a given pair of the closely-spaced parallel runway is carried out according to the traffic scenario shown in Figure 5. 


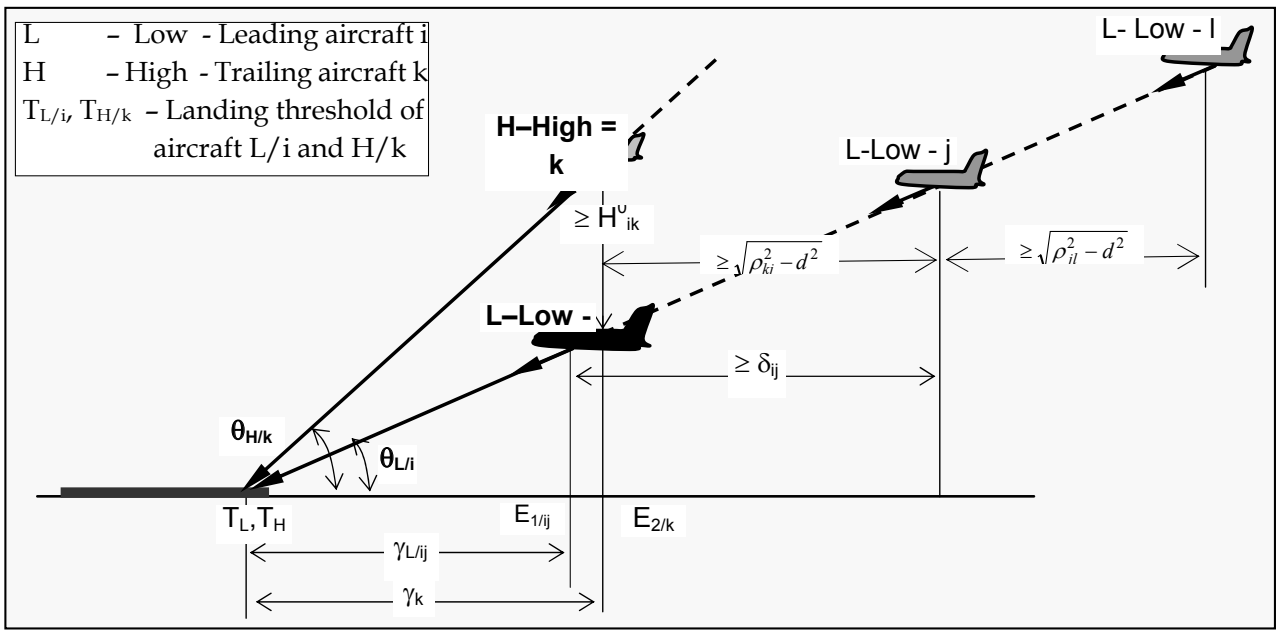

Fig. 5. The geometry of CNAP and SEAP in the vertical plane applied to the closely spaced parallel runways under IMC (Compiled from: Janic, 2008a)

As can be seen, as in Figure $4 b$, the aircraft (i), as the leading one in the pair $(i k)$ and the sequence $(i j)$, approaches to the ultimate RWY1. The aircraft $(k)$ as the trailing in the pair $(i k)$ approaches to the ultimate RWY2 (Janic, 2006). Thus, the pair of aircraft (ij) is going to land on RWY1 and the aircraft $(k)$ on RWY2. The order of landings on either runway is $(i, k, j)$. This implies that the pair (ij) is influenced by the aircraft $(k)$. Another pair $(k l)$ in Figure 5 is influenced by the aircraft $(j)$.

\subsubsection{The minimum inter-arrival times at the "reference location(s)"}

The inter-arrival times at $t_{j / k}$ for particular "strings" of landing aircraft (ijk) in Figure 5 are calculated under assumption that each aircraft category can perform both CNAP and SEAP (Janic, 2006, 2008b). Regarding the relative speeds along the final approach trajectories, the aircraft (ikj) can relate to each other as either "fast" $F$ or "slow" $S$, which gives eight combinations. In the first four, the aircraft (i) and (j) are considered as either "slow" $S$ or "fast" $F$; the aircraft $(k)$ is considered as "slow" $S$. The possible combinations of sequences are: $S-S-S$, $S-S-F, F-S-S$ and $F-S-F$. In other four combinations, the aircraft $(k)$ is considered as "fast" $F$. The possible combinations of sequences are: $S-F-S, S-F-F, F-F-S$ and $F-F-F$. After selecting the control variable $u$, the attributes "low" $L$ and "high" $H$ can be additionally attached to each aircraft in each of the above-mentioned landing sequences. One of the principles can be that in any sequence, the "slow" aircraft always performs SEAP (i.e. as "high" $H$ ) and the "fast" aircraft always performs CNAP (i.e. as "low" L). The same applies to the aircraft "string" ( $\mathrm{kjl}$ ). In developing expressions for calculating the minimum inter-arrival times $a_{i j / k}$ the following notation is used: the aircraft $(k)$ landing on RWY2, respectively; 
$d \quad$ is spacing between centerlines of the closely-spaced parallel runways;

$v_{i / k j} \quad$ is the final approach speed of the aircraft $(i),(k)$ and $(j)$, respectively;

$\theta_{i / k / j} \quad$ is the GS angle of trajectory of the aircraft $(i),(k)$ and $(j)$, respectively;

$\delta_{i j} \quad$ is the ATC minimum longitudinal (in-trail) separation rules applied to the aircraft pair (ij);

$\rho_{i k / k j} \quad$ is the ATC minimum horizontal-diagonal separation rules applied to the aircraft pairs $(i k)$ and $(k j)$, respectively;

is the ATC minimum vertical separation rules applied to the aircraft pairs $(i j),(i k)$

$H^{0} i / k j j$ and $(k j)$, respectively;

$u_{i j}$ is the control variable taking the value " 0 " if the ATC longitudinal (in-trail)

$u_{i k}$ separation rules between the aircraft pair (ij) and the ATC horizontal-diagonal

$u_{k j} \quad$ separation rules between the aircraft pair $(i k)$ and $(k j)$ are applied, respectively, and the value " 1 ", otherwise, i.e. if the ATC vertical separation rules between aircraft in given pairs are applied, respectively.

$u_{k j}$ is the control variable taking the value " 0 " if the ATC longitudinal (in-trail) $u_{j l}, u_{k l}$ separation rules between the aircraft $(k l)$ and the ATC horizontal-diagonal separation rules between the aircraft pairs $(k j)$ and $(j l)$ are applied, respectively, and the value " 1 ", otherwise, i.e., if the ATC vertical separation rules between aircraft in given pairs are applied, respectively.

Respecting the approach procedures (CNAP and SEAP) and the associated ATC separation rules for different combinations of aircraft landing sequences, expressions for the minimum times ${ }_{a} t_{i j / k}$ are developed as follows (Janic, 2009).

i) Sequences $v_{i} \leq v_{k} \leq v_{i}$; Aircraft speed/procedure combination: $S / H-S / H-S / H, S / H-S / H-F / L$, $S / H-F / L-F / L, F / L-F / L-F / L$

The aircraft $(i),(k),(j)$ are separated by the ATC minimum separation rules at the moment when the aircraft (i) arrives at the landing threshold of RWY1. The inter arrival time ${ }_{a} t_{i j / k}$ is determined as follows:

$$
{ }_{a} t_{i j / k}={ }_{a} t_{i k}+{ }_{a} t_{k j}=\max \left\{\begin{array}{l}
\left(1-u_{i j}\right) \delta_{i j} / v_{j}+u_{i j} H_{i j}^{0} / v_{j} \sin \theta_{j} ; \\
\left(1-u_{i k}\right)\left(\sqrt{\rho_{i k}^{2}-d^{2}} / v_{k}\right)+u_{i k}\left(H_{i k}^{0} / v_{k} \sin \theta_{k}\right)+ \\
\left(1-u_{k j}\right)\left(\sqrt{\rho_{k j}^{2}-d^{2}} / v_{j}\right)+u_{k j}\left(H_{k j}^{0} / v_{j} \sin \theta_{j}\right)
\end{array}\right\}
$$


In expression $(7 \mathrm{a})$, the aircraft $(i)$ and $(k)$ perform SEAP $\left(u_{i k}=1\right)$ and the aircraft $(j)$ performs CNAP, i.e. $u_{i j}=u_{k j}=0$; in addition $u_{j l}=1$ if the aircraft $(l)$ of the pair $(j l)$ is $F / L$, and $u_{j l}=0$ if it is $S / H$; consequently $u_{k l}=u_{k j}=0$.

\section{ii) Sequence: $v_{i}>v_{k} \geq v_{i:}$ Aircraft speed/procedure combination: F/L-S/H-S/H}

The aircraft $(i k)$ and $(k j)$ are separated by the ATC minimum separation rules at the moment when the leading aircraft (i) is at FAG of RWY1. The inter arrival time ${ }_{a} t_{i j / k}$ is determined as follows:

$$
{ }_{a} t_{i j / k}={ }_{a} t_{i k}+{ }_{a} t_{k j}=\max \left\{\begin{array}{l}
\left(1-u_{i j}\right)\left[\delta_{i j} / v_{j}+\left(\gamma_{j} / v_{j}-\gamma_{i} / v_{i}\right)\right]+ \\
u_{i j}\left[H_{i j}^{0} / v_{j} \sin \theta_{j}+\gamma_{i} \sin \theta_{i}\left(1 / v_{j} \sin \theta_{j}-1 / v_{i} \sin \theta_{i}\right)\right] ; \\
\left(1-u_{i k}\right)\left(\sqrt{\rho_{i k}^{2}-d^{2}} / v_{k}+\gamma_{k} / v_{k}-\gamma_{i} / v_{i}\right)+ \\
u_{i k}\left[H_{i k}^{0} / v_{k} \sin \theta_{k}+\gamma_{i} \sin \theta_{i}\left(1 / v_{k} \sin \theta_{k}-1 / v_{i} \sin \theta_{i}\right)\right]+ \\
\left(1-u_{k j}\right)\left(\sqrt{\rho_{k j}^{2}-d^{2}} / v_{j}+\gamma_{j} / v_{j}-\gamma_{k} / v_{k}\right)+ \\
u_{k j}\left[H_{k j}^{0} / v_{j} \sin \theta_{j}+\gamma_{k} \sin \theta_{k}\left(1 / v_{j} \sin \theta_{j}-1 / v_{k} \sin \theta_{k}\right)\right]
\end{array}\right\}
$$

In expression $(7 \mathrm{~b})$ the aircraft $(i)$ performs CNAP and the aircraft $(k)$ and $(j)$ perform SEAP, i.e., $u_{i j}=u_{i k}=u_{k j}=0$; in addition $u_{j l}=1$ if the aircraft $(l)$ is $F / L$ and $u_{j l}=0$ if it is $S / H$; consequently in both cases $u_{k l}=u_{k j}$.

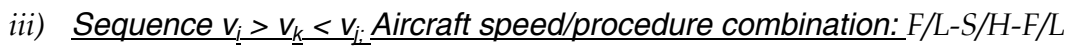

The aircraft $(i k)$ are separated by the ATC minimum separation rules at the moment when the leading aircraft $(i)$ is at FAG of RWY1. The aircraft in the pair $(k j)$ are separated by the ATC minimum separation rules at the moment when the aircraft $(k)$ arrives at the landing threshold of RWY2. The inter arrival time $a_{i j / k}$ is determined as follows:

$$
{ }_{a} t_{i j / k}={ }_{a} t_{i k}+{ }_{a} t_{k j}=\max \left\{\begin{array}{l}
\left(1-u_{i j}\right) \delta_{i j} / v_{j}+u_{i j} H_{i j}^{0} / v_{j} \sin \theta_{j} ; \\
\left(1-u_{i k}\right)\left(\sqrt{\rho_{i k}^{2}-d^{2}} / v_{k}+\gamma_{k} / v_{k}-\gamma_{i} / v_{i}\right)+ \\
u_{i k}\left[H_{i k}^{0} / v_{k} \sin \theta_{k}+\gamma_{i} \sin \theta_{i}\left(1 / v_{k} \sin \theta_{k}-1 / v_{i} \sin \theta_{i}\right)\right] \\
+\left(1-u_{k j}\right)\left(\sqrt{\rho_{k j}^{2}-d^{2}} / v_{j}\right)+u_{k j} H_{k j}^{0} / v_{j} \sin \theta_{j}+
\end{array}\right\}
$$

In expression (7c), the aircraft $(i)$ and $(j)$ perform CNAP and the aircraft $(k)$ performs SEAP, i.e., $u_{i j}=u_{k j}=0$ and $u_{i k}=1$; in addition $u_{j l}=1$ if the aircraft $(l)$ is $F / L$ and $u_{j l}=0$ if it is $S / H$; consequently in both cases $u_{k l}=u_{k j}$. 


\section{iv) Sequences $v_{i}=v_{k}>v_{j}$. Aircraft speed/procedure combination: $F / L-F / L-S / H$}

The aircraft $(i k)$ are separated by the ATC minimum separation rules at the moment when the aircraft $(i)$ is at FAG and further when it arrives at the landing threshold of RWY1. The aircraft $(k j)$ are separated by the ATC minimum separation rules at the moment when the aircraft $(k)$ is at the final approach gate of RWY2. The inter arrival time ${ }_{a} t_{i j / k}$ is determined as follows:

$$
{ }_{a} t_{i j / k}={ }_{a} t_{i k}+{ }_{a} t_{k j}=\max \left\{\begin{array}{l}
\left(1-u_{i j}\right)\left[\delta_{i j} / v_{j}+\left(\gamma_{j} / v_{j}-\gamma_{i} / v_{i}\right)\right]+ \\
u_{i j}\left[H_{i j}^{0} / v_{j} \sin \theta_{j}+\gamma_{i} \sin \theta_{i}\left(1 / v_{j} \sin \theta_{j}-\gamma_{i} / v_{i} \sin \theta_{i}\right)\right] ; \\
\left(1-u_{i k}\right)\left(\sqrt{\rho_{i k}^{2}-d^{2}} / v_{k}\right)+u_{i k}\left(H_{i k}^{0} / v_{k} \sin \theta_{k}\right)+ \\
\left(1-u_{k j}\right)\left(\sqrt{\rho_{k j}^{2}-d^{2}} / v_{j}+\gamma_{j} / v_{j}-\gamma_{k} / v_{k}\right)+ \\
u_{k j}\left[H_{k j}^{0} / v_{j} \sin \theta_{j}+\gamma_{k} \sin \theta_{k}\left(1 / v_{j} \sin \theta_{j}-1 / v_{i} \sin \theta_{k}\right)\right]
\end{array}\right\}
$$

In expression (7d), the aircraft $(i)$ and $(k)$ perform CNAP and the aircraft $(j)$ performs SEAP, i.e., $u_{i j}=u_{k j}=1$ and $u_{i k}=0$; in addition $u_{j l}=1$ if the aircraft $(l)$ is $F / L$ and $u_{j l}=0$ if it is $S / H$; consequently in both cases $u_{k l}=u_{k j}$.

\section{v) Sequences $v_{i}<v_{\underline{k}}>v_{j}$. Aircraft speed/procedure combination: $S / H-F / L-S / H$}

The aircraft $(i k)$ are separated by the ATC minimum separation rules at the moment when the aircraft $(i)$ arrives at the landing threshold of RWY1. The aircraft $(k j)$ are separated by the ATC minimum separation rules at the moment when the aircraft $(k)$ is at the final approach gate of RWY2. The inter arrival time ${ }_{a} t_{i j / k}$ is determined as follows:

$$
{ }_{a} t_{i j / k}={ }_{a} t_{i k}+{ }_{a} t_{k j}=\max \left\{\begin{array}{l}
\left(1-u_{i j}\right) \delta_{i j} / v_{j}+u_{i j} H_{i j}^{0} / v_{j} \sin \theta_{j} ; \\
\left(1-u_{i k}\right)\left(\sqrt{\rho_{i k}^{2}-d^{2}} / v_{k}+u_{i k}\left(H_{i k}^{0} / v_{k} \sin \theta_{k}\right)+\right. \\
\left(1-u_{k j}\right)\left(\sqrt{\rho_{k j}^{2}-d^{2}} / v_{j}+\gamma_{j} / v_{j}-\gamma_{k} / v_{k}\right)+ \\
u_{k j}\left[H_{k j}^{0} / v_{j} \sin \theta_{j}+\gamma_{k} \sin \theta_{k}\left(1 / v_{j} \sin \theta_{j}-1 / v_{i} \sin \theta_{k}\right)\right]
\end{array}\right\}
$$

In expression (7e), the aircraft $(i)$ and $(j)$ perform SEAP and the aircraft $(k)$ performs CNAP, i.e., $u_{i j}=u_{k j}=1$ and $u_{i k}=0$; in addition $u_{j l}=1$ if the aircraft $(l)$ is $F / L$ and $u_{j l}=0$ if it is $S / H$; consequently in both cases $u_{k l}=u_{k j}$.

Expression 7(a-e) can then be used in combination with expression (3) to calculate the landing capacity of a given pair of the closely-spaced parallel runways from expression (4). 


\section{An application of the methodology}

\subsection{Background}

The application of the above-mentioned methodology for assessment of the potential of some innovative operational procedures to increasing the airport runway landing capacity is carried out by applying particular models to the generic and the specific airport runway case using the "what-if" scenario approach (Janic, 2006, 2008, 2008a, 2009).

\subsection{The ATC time-based separation rules}

The model of the "ultimate" capacity of a single runway using the ATC time-based instead of the ATC distance-based separation rules for landing aircraft is applied using the generic input. This relates to the size (i.e. geometry) of the "wake reference airspace", characteristics of the wake vortices of the landing aircraft fleet, behavior of the wake vortices within and around the "wake reference airspace" influenced by the external weather conditions, and the current ATC distance-based separation rules.

\subsubsection{Input}

\subsubsection{The size of the "wake reference airspace"}

The size of the "wake reference airspace" is determined by using the following input: The length of the common approach path between FAG and the runway landing threshold $T$ in Figure 3 is taken to be similar to that at most airports, i.e. $\gamma=6 \mathrm{~nm}$. Since the aircraft use ILS, the distance from the threshold to the ultimate point of touchdown is assumed to be $\Delta=0.16 \mathrm{~nm}$, i.e. $300 \mathrm{~m}$. This gives the total distance between FAG and the runway touchdown of $6.16 \mathrm{~nm}$. The nominal ILS GS angle is $\theta=3^{0}$ with the maximum deviations of about $\pm 0.5^{0}$. The angle between the axis and each side of the "wake reference airspace" in the horizontal plane is determined by the characteristics of the ILS LLZ (Localizer) and amounts to $\alpha= \pm 1.5^{\circ}$. The distance between the ILS Outer Marker (OM) and the landing threshold $T$ is $\beta=4 n m$. Consequently, the "wake reference profiles" along the "wake reference airspace" are calculated depending on the distances and times from the landing threshold and given in Table 2.

\begin{tabular}{|c|c|c|}
\hline $\begin{array}{l}\text { Distance/time } \\
\text { to the landing }\end{array}$ & \multicolumn{2}{|c|}{ The size of the profile } \\
\hline$(\mathrm{nm}) /(\mathrm{s})^{1)}$ & $\underset{(\mathrm{ft})}{\mathbf{y}}$ & $\frac{\underline{\mathbf{Z}}}{(\mathrm{ft})}$ \\
\hline $6 / 0$ & 2000 & 600 \\
\hline $5 / 27$ & 1600 & 500 \\
\hline $4 / 54$ & 1200 & 400 \\
\hline $3 / 81$ & 950 & 300 \\
\hline $2 / 108$ & 640 & 200 \\
\hline $0 / 162$ & 200 & 50 \\
\hline
\end{tabular}

Table 2. The size of the "wake free profile" depending on distance and time to the landing threshold 


\subsubsection{Characteristics of the aircraft fleet}

In this case, the aircraft types are categorized into four categories following Table 1 . Their average characteristics, based on the specific values of particular parameters including the calculated wake vortex parameters of particular category, are given in Table 3.

\begin{tabular}{cccccc}
$\begin{array}{c}\text { Aircraft } \\
\text { category }\end{array}$ & Mass & Wing span & Approach speed & Circulation & $\begin{array}{c}\text { The wake } \\
\text { reference time }\end{array}$ \\
& $\underline{\mathbf{M}}$ & $\underline{\mathbf{B}}$ & $\begin{array}{c}\mathbf{v} \\
(\mathrm{kts})^{1)}\end{array}$ & $\begin{array}{c}\Gamma_{0} \\
\left(\mathrm{~m} / \mathrm{s}^{2}\right)^{2}\end{array}$ & $\begin{array}{c}\mathbf{t}^{*} \\
\left.(\mathrm{~s})^{2}\right)\end{array}$ \\
\hline Small & 20 & 24 & $120 / 90$ & $138 / 184$ & $16 / 12$ \\
Large & 55 & 30 & $140 / 120$ & $260 / 303$ & $13 / 12$ \\
B757 & 117 & 38 & $170 / 140$ & $359 / 436$ & $16 / 13$ \\
Heavy & 206 & 65 & $170 / 140$ & $370 / 449$ & $44 / 36$ \\
\hline
\end{tabular}

1) The maximum and the minimum approach speed, respectively, at FAG and the landing threshold $\mathrm{T}, 2)$ The values correspond to the maximum and the minimum approach speed, respectively. Compiled from (NASA 1999, 2001; Donohue and Rutishauser, 2001)

Table 3. Characteristics of the particular aircraft landing categories (the averages)

In addition, the initially generated wake vortices are assumed to decay to the observed typical atmospheric background circulation of $\Gamma^{*}=70 \mathrm{~m}^{2} / \mathrm{s}$ over the period $k=8 t^{*}$ (Donohue and Rutishauser, 2001 Sarpkaya, 2000; Shortle and Jeddi, 2007). The proportion of particular aircraft categories in the aircraft fleet mix is varied parametrically.

\subsubsection{The external conditions}

The external conditions are specified by a constant crosswind of $V_{c w}=5 \mathrm{~m} / \mathrm{s}$, which is above the conditions of "no wind" of $V_{c w} \leq 3 \mathrm{~m} / \mathrm{s}$. The influence of the headwind $V_{h w}(t)$ is not particularly considered since some preliminary calculations have shown that even a very strong headwind cannot increase the vertical distance between the wake vortex of the leading and the flight path of the trailing aircraft in a shorter time than that obtained by the current ATC distance-based separation rules.

\subsubsection{The ATC separation rules}

The ATC minimum distance-based separation rules in Table 1 are used as the basis for initial setting up the ATC time-based separation rules in combination with the average runway landing occupancy time of $t_{a i}=60 \mathrm{~s}$ for all aircraft categories.

\subsubsection{Results}

The results from the model application consist of the following components:

- The strength (i.e. circulation) of wake vortices to which the trailing aircraft are exposed in particular landing sequences if the ATC VFR and IFR in Table 1 are applied; 
- The matrix of the standardized time-based separation rules for particular categories of the aircraft landing sequences; and

- The runway landing capacity calculated for the current ATC distance-based VFR and IFR separation rules, and the ATC tiem-based separation rules based on the wake vortex behavior influenced by weather (wind) conditions.

The strength (i.e. circulation) of the wake vortices to which the trailing aircraft in particular landing sequences are potentially exposed when the minimum ATC IFR and VFR are applied is given in Table 4.

ATC VFR

\begin{tabular}{ccccc}
\hline$\underline{i} \boldsymbol{j} j$ & Small & Large & B757 & Heavy \\
\hline Small & 134 & 134 & 134 & 134 \\
Large & 207 & 231 & 231 & 231 \\
B757 & 244 & 275 & 305 & 313 \\
Heavy & 317 & 333 & 379 & 379 \\
\hline i/j & Small & ATC IFR & & Heavy \\
\hline Small & 17 & 62 & B757 & 69 \\
Large & 0 & 87 & 69 & 101 \\
B757 & 0 & 70 & 101 & 79 \\
Heavy & 181 & 234 & 79 & 197 \\
\hline
\end{tabular}

Table 4. The potential circulation $\Gamma(t)$, which the trailing aircraft faces under the ATC VFR and IFR while flying at the given approach speeds (see Table 3 )

As can be seen the potential wake vortex strength is higher under VFR than under IFR as could be intuitively expected. In addition, in both cases, for most sequences this circulation is significantly higher than the typical atmospheric circulation of $70 \mathrm{~m}^{2} / \mathrm{s}$. Furthermore, it should be born in mind that the trailing aircraft of different types in the particular sequences are sensitive differently to the different strength of the wake vortices. Last but not least, the trailing aircraft are not actually exposed to such circulation because the wakes of the leading aircraft sink below their flight paths thanks to their self-induced descent speed simultaneously with their decaying. This again illustrates the fact that the landing aircraft could also be put closer to each other under IMC just as under the VMC without significant risk of the wake vortex hazard, but, of course, only if the corresponding technology for "see and be seen" was available under IMC. In such case, the separation rules under IMC and VMC would be unified as the ATC time-based separation rules. The basis for setting up these rules would be the existing ATC VFR (Table 1) and the typical aircraft approach speeds (Table 3). Table 5 gives an example of such standardized time-based separation rules. 


\begin{tabular}{ccccc}
\hline i/j & Small & Large & B757 & Heavy \\
\hline Small & 1.0 & 1.0 & 1.0 & 1.0 \\
Large & 1.5 & 1.0 & 1.0 & 1.0 \\
B757 & 2.0 & 1.3 & 1.5 & 1.2 \\
Heavy & 2.5 & 1.5 & 1.5 & 1.2 \\
\hline
\end{tabular}

Table 5. The standardized ATC time-based minimum VFR/IFR separation rules $\tau_{i j / m i n}$ for landing aircraft (min)

The particular values in Table 5 are rounded-up in order to be convenient for practical use. As can be seen, in some landing sequences, the runway landing occupancy times can be used as the minimum separation rule. In addition, as on case of the distance-based rules, these rules are applied depending on the landing sequence at the runway threshold $\left(v_{i} \leq v_{j}\right)$ and, at FAG $\left(v_{i}>\right.$ $\left.v_{j}\right)$.

Using the above-mentioned inputs in Tables 3 and 5, the runway landing capacity is calculated for different cases. Figure 6 shows the dependence of this capacity on the proportion of Heavy aircraft in the fleet, the above-mentioned separation rules, and the wake vortex characteristics and behavior. The proportion of Small and B757 aircraft is kept constant, each of 5\%.

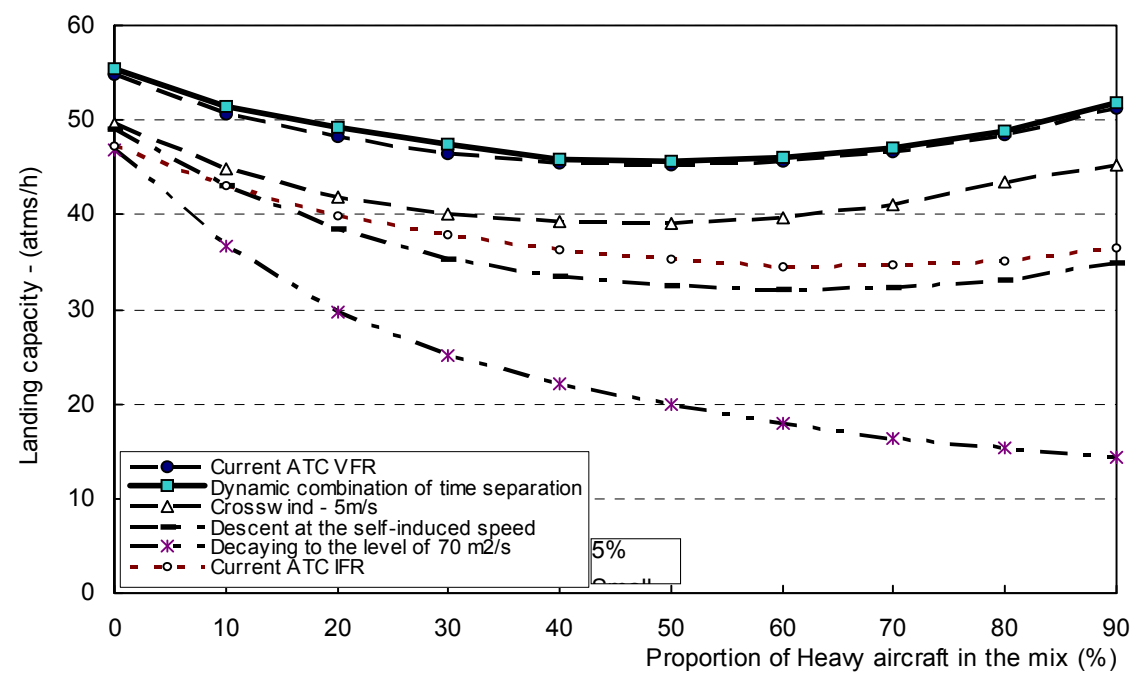

Fig. 6. Dependence of the runway landing capacity on the aircraft fleet mix, the ATC separation rules, and the wake vortex characteristics and behavior

As can be seen, in the case when the ATC time separation rules are based on the wake decaying to the typical atmospheric circulation of $70 \mathrm{~m}^{2} / \mathrm{s}$, the capacity is the lowest and continuously decreases with increasing the proportion of Heavy aircraft in the mix, as compared with the other cases. The latter is because the stronger wakes of the leading Heavy aircraft need a longer 
time to decay to the safe level. In other cases the capacity decreases with increasing of the proportion of Heavy aircraft in the mix up to about $20 \%$, and then increases again. In the former case, the impact of strong wakes behind Heavy aircraft prevails. In the latter case, the higher approach speeds of Heavy aircraft prevail.

In addition, the capacity for the nominal ATC VFR is higher than the capacity for the nominal ATC IFR by about $30 \%$ as shown in Figure 2. If the time-based separation rules were applied under the conditions of a crosswind of $5 \mathrm{~m} / \mathrm{s}$, the capacity would be somewhere in between the current VFR and IFR capacity. This indicates that the capacity gains would be comparable to the IFR capacity if the influence of the crosswind on the wake vortex behavior was taken into account. When the time-based separation rules respecting the wake vortex descent time, were applied the corresponding capacity would be lower than the current IFR capacity. This implies that the current IFR seem to be based only partially on the descent time of the wake vortices below the flight path of the trailing aircraft and not on the time they need to completely move out of the "wake reference airspace".

The dynamically selected ATC time-based separation rules for particular landing sequences combining the current ATC VFR and the ambient factors influencing the wake vortex behavior seem to be able to produce the highest capacity. However, in the given example, this capacity would only be just slightly higher than the capacity obtained under the current ATC VFR. This again suggests that the current ATC VFR could be the basis for setting up the corresponding time-based separation rules, which would be also applicable under both VMC and IMC, thus stabilizing the runway landing capacity with respect to changes of weather.

\subsection{The Steeper Approach Procedure (SEAP)}

\subsubsection{The case of San Francisco international Airport (U.S.)}

\subsubsection{Inputs}

The model for estimating the landing capacity of the closely-spaced parallel runways when both CNAP and SEAP are used has been applied to the traffic scenario at San Francisco International Airport (SFO) (U.S.) shown in Figure 7 (Janic, 2008a). 


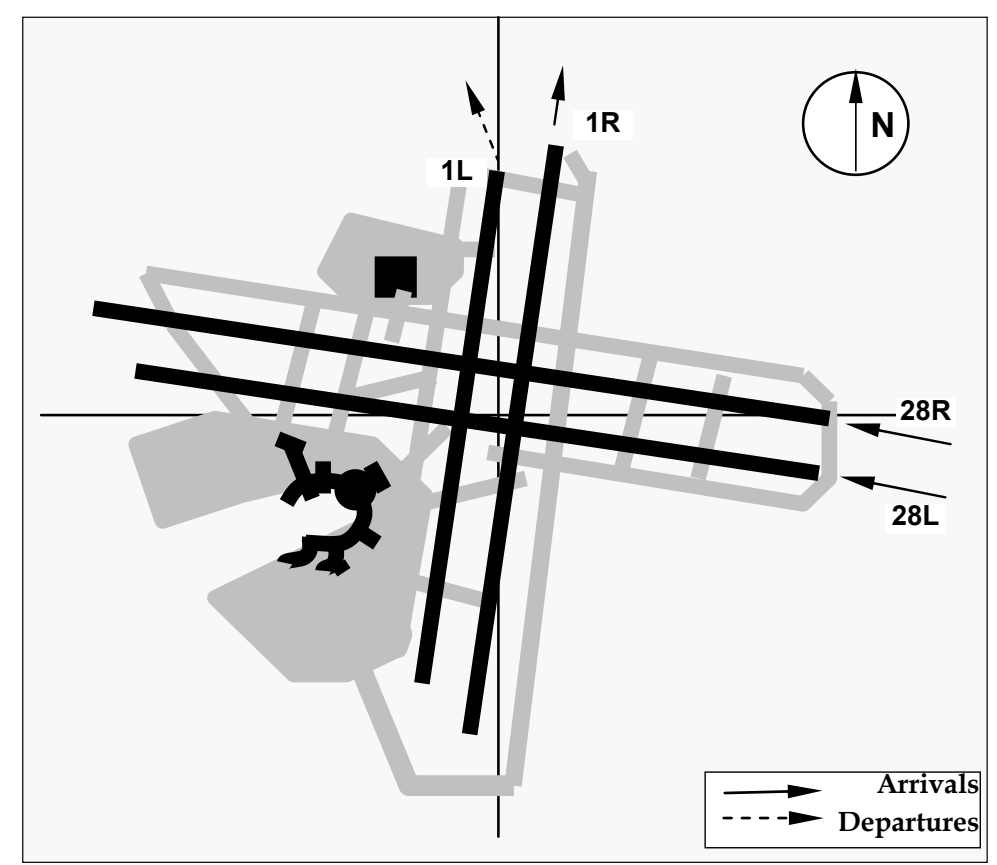

Fig. 7. Simplified layout of the runway system at San Francisco International Airport (SFO) (Compiled from Janic, 2008a)

Currently, the airport operates two pairs of the closely-spaced parallel runways - $1 \mathrm{~L} / \mathrm{R}$ and $28 \mathrm{R} / \mathrm{L}$. Dimensions of runways $1 \mathrm{~L} / 28 \mathrm{R}$ are $11879 \times 200 \mathrm{ft}(3618 \times 61 \mathrm{~m})$. Dimensions of the runways $1 \mathrm{R} / 28 \mathrm{~L}$ are $10602 \times 200 \mathrm{ft}(3231 \times 61 \mathrm{~m})$. Each pair of runways is spaced for $750 \mathrm{ft}$ (i.e., $228.75 \mathrm{~m}$ ), which is sufficient for simultaneous arrivals and departures under VMC but not under IMC (FAA, 2006). Under preferable VMC the runways 28R/L are used for paired arrivals and the runways $1 \mathrm{~L} / \mathrm{R}$ for paired departures as shown in Figure 7 . In dependence on the mixture of arrivals and departures, the paired departures can be realized between the successive pairs of arrivals. When the weather deteriorates due the specified minima in Figure 1 each pair of runways is used as the single runway for both arrivals and departures (West Plan for Runways 28R/L and Southeast Plan for Runways 1L/R). Such bad weather prevails on average for about $20 \%$ of time during the year (Cotton et al., 20011). In addition to geometry of runways, the additional inputs for the model are as follows: The arrivals are realized on the runways $28 \mathrm{R} / \mathrm{L}$ or $1 \mathrm{~L} / \mathrm{R}$ with the length of final approach path, i.e., the distance between FAG and landing threshold(s), $E_{L / 1}$ and $E_{H / 2}$ and the runway thresholds $T_{L / 1}$ and $T_{H / 2}$ of $\gamma_{H / 1}=\gamma_{L / 2}=12$ $n m$ (see Figure 5). If the standard GS angle $\theta_{l}=3^{0}$ is used the aircraft altitude at the gate $E_{1 / 2}$ will be $4000 \mathrm{ft}$; if the steeper GS angle $\theta_{H}=5.5^{\circ}$ is used, this altitude will be about $7000 \mathrm{ft}$. The difference is greater than the ATC minimum vertical separation rules (1000ft) thus enabling safe pairing of aircraft of particular wake vortex/speed combinations approximately above each other at the entry gates $E_{L / 1}$ and $E_{H / 2}$, respectively (FAA, 2006).

The aircraft fleet in terms of the weight and wake vortex categories is as follows: Small $(7 \%)$. Large (52\%), B757 (19\%), and Heavy (22\%). The average approach speeds of particular aircraft categories are as follows: Small 120kts, Large 130kts, B757 140kts, and Heavy 150kts. The 
average runway landing occupancy time is 40s for Small and 50s for Large, B757 and Heavy. The average departure runway occupancy time is 30s for Small, and 40s for Large, B757 and Heavy aircraft categories (LMI, 2004).

The ATC is supposed to apply the minimum longitudinal and horizontal-diagonal radar-based separation rules between arriving aircraft similarly as given in Table 1. The radar-based minimum horizontal-diagonal separation rules are: $\rho=2.5 \mathrm{~nm}$. The minimum vertical separation rules are: $H=1000 \mathrm{ft}$. The separation between the arrival and departure on the same runway is ${ }_{d} \delta_{(.)}=2 n m($ FAA, 2006; NASA, 1998).

Assignment of the approach procedures (CNAP or SEAP) to particular aircraft categories is carried out according to the two hypothetical scenarios: Scenario 1 implies that only Small aircraft can perform SEAP. Scenario 2 implies that Small, Large and B757 aircraft can perform SEAP. In both Scenarios Heavy aircraft do not perform SEAP. Comparing to the present situation the former Scenario looks more realistic than the latter.

\subsubsection{Results}

Using the above-mentioned inputs has enabled calculation of the "ultimate" landing capacity of the closely-spaced parallel runways for eth case airport - SFO. In addition, the "ultimate" capacities for take-offs and mixed operations are calculated in order synthesize the capacity coverage curve(s). The results are shown in Figure 8.

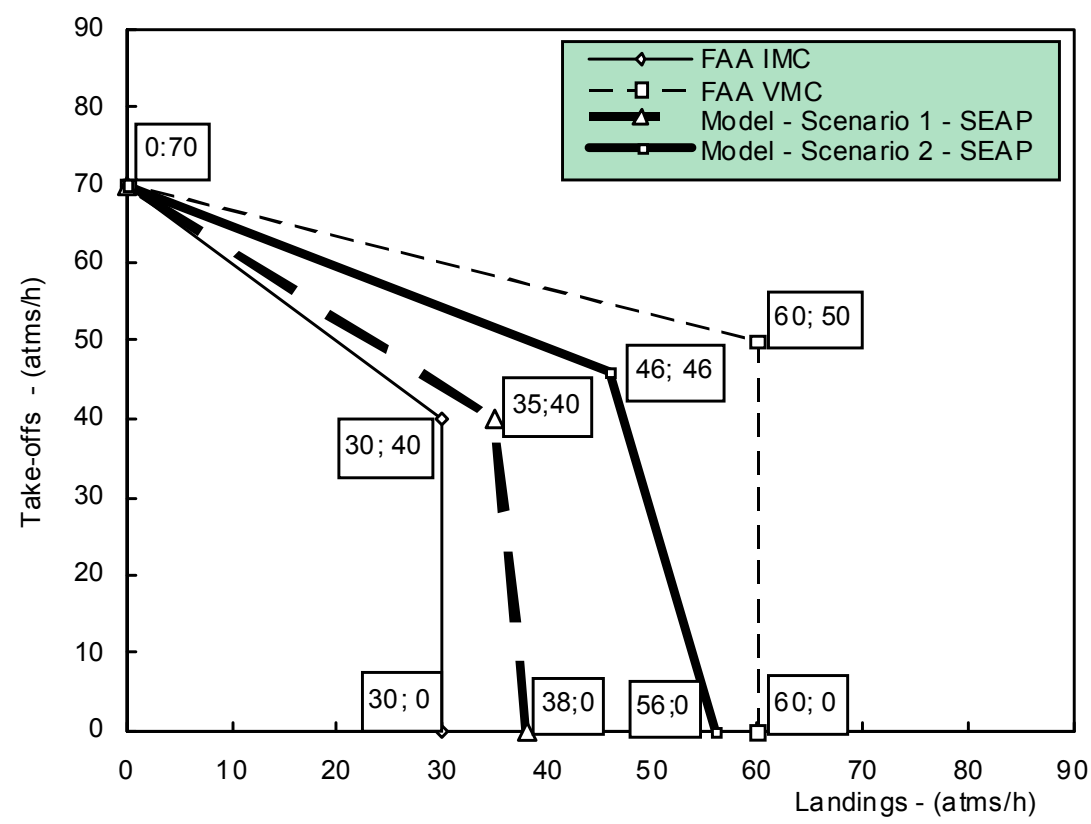

Fig. 8. The capacity of San Francisco International Airport achieved by different approach procedures (Compiled from Janic, 2008a) 
The VMC and IMC capacity curves for the current situation are synthesized from the FAA airport capacity benchmark calculations (FAA, 2004a). As can be seen, according to Scenario 1 in which only Small aircraft performed SEAP, the arrival capacity would be 38 arr $/ \mathrm{h}$, the mixed capacity $35 \mathrm{arr} / \mathrm{h}$ and $35 \mathrm{dep} / \mathrm{h}$, and the departure capacity $70 \mathrm{dep} / \mathrm{h}$. This is higher than the corresponding current benchmarking capacities for about $27 \%, 17 / 5 \%$ and $0 \%$, respectively. According to Scenario 2 when all except Heavy aircraft performed SEAP, the arrival capacity would be $56 \mathrm{arr} / \mathrm{h}$, the mixed capacity $46 \mathrm{arr} / \mathrm{h}$ and $46 \mathrm{dep} / \mathrm{h}$, and the departure capacity 70 $\mathrm{dep} / \mathrm{h}$. These are for about $83 \%, 53 \% / 13 \%$, and $0 \%$, respectively, higher than the current corresponding IMC capacities when CNAP is performed. As well, these capacities are for about $7 \%, 30 / 9 \%$, and $0 \%$ lower than the current corresponding VMC capacities, respectively. This indicates that in given case, the SEAP could have the potential to rather substantively increase the "ultimate" (landing) capacity of the given system of closely-spaced parallel runways under IMC and IFR (FAA, 2004a; Janic, 2008a).

\section{Conclusions}

This Chapter has presented the potential of some innovative procedures for increasing the airport runway landing capacity. These have included: i) the ATC tine-based separation rules between landing aircraft on a single runway and ii) the SEAP (Steeper Approach Procedure) to the closely-spaced parallel runways. The methodology consisting of the dedicated models of the "ultimate" runway capacity under the above-mentioned conditions has been developed and applied.

In particular, the model of the landing capacity based on the ATC time-based separation rules has been applied to a busy landing runway with the given geometry of the "wake reference airspace" serving the four aircraft FAA/ICAO categories. These have been characterized by the wake vortex parameters (the approach speed, the wing span, and weight), and the runway landing occupancy time under given atmospheric (crosswind) conditions. The results have indicated that the ATC time-based separation rules, based exclusively on the wake vortices decaying to the typical atmospheric circulation, have produced the lowest runway landing capacity. The dynamically selected ATC time-based separation rules based on the current ATC VFR and the influence of the crosswind on the wake vortices have produced the highest runway landing capacity. The ATC time separations based on the wake vortex self-induced descent speed have produced a landing capacity slightly lower than the capacity achieved under the current ATC IFR. Finally, the ATC time-based separation rules based on the impact of the crosswind on the wake vortices have produced a capacity, which is somewhere between the capacities achieved under the current distance-based VFR and the IFR. In all cases, the landing capacity has generally decreased with increasing of the heterogeneity of the aircraft fleet mix and particularly with increasing of the proportion of Heavy aircraft in the fleet mix.

The model for the landing capacity of the closely-spaced parallel runways when both CNAP (Conventional Approach Procedure) and SEAP (Steeper Approach Procedure) are simultaneously used has been applied to the traffic scenario of San Francisco International (SFO) airport (US). The results have indicated that SEAP as compared to CNAP has possessed the potential for increasing the IMC landing capacity of given parallel runways for about $27 \%$ when only the small aircraft could perform SEAP, and for about $83 \%$ when all except Heavy aircraft could perform SEAP. Consequently a gap between the current VMC and IMC landing capacity could be narrowed to about $7 \%$. 
In general, the SEAP has shown advantages in comparison to the current CNAP as follows: i) significant increase in the IMC runway landing capacity; ii) substantive filling in existing gap between VMC and IMC landing capacity; and iii) diminished sensitivity of the landing capacity to the weather conditions, thus making the airport and airline operations more reliable and predictable.

\section{References}

Andreatta, G., Romanin-Jacur, G., (1987), "Aircraft Flow Management under Congestion", Transportation Science, Vol. 21, No. 4, 249-253

Bianco, L. Bielli, M., (1992), "Air Traffic Management: Optimization Models and Algorithms", Journal of Advanced Transportation, Vol. 26, No. 2, 1992, 131-167

Blumstein, A., (1959), “The Landing Capacity of a Runway”, Operations Research, Vol. 7, No.1, 1959, pp. 752-763

Burnham, D. C., Hallock, J. N., Greene, G. C., (2002), “Wake Turbulence Limits on Paired Approaches to Parallel Runways", Journal of Aircraft, Vol. 39, No. 4, pp 630-637

Choroba, P., (2003), “A Vision of Wake Vortex Research for Next 20 Years”, Working paper, EUROCONTROL, Bretigny sur de Orge, Paris, France

Cotton, W., Foggin, J., Gosling, G. D., (2001), Potential Future Contribution of Air Traffic Management Technology to the Capacity of San Francisco International Airport, Report on the Independent Technology Panel, Federal Aviation Administration EIR/EIS-Emerging \& New Technologies, San Francisco, California, USA

CRS, (2007), Aviation Congestion and Delay: System-Wide and New York Area Issues, CRS Report for Congress, Congressional Research Service, RL 34284, Washington DC, USA

Czerny, A. I., Forsyth, P., Gillen, D., Niemeier, H. M., (2008), Airport Slots: International Experiences and Options for Reform, Ashgate Publishing Limited, Aldershot, UK

Donohue, G. L., (1999), “A Simplified Air Transportation Capacity Model”, Journal of ATC, April-June, pp. 8-15

Donohue, G. L., Rutishauser, D. K., (2001) “The Effect of Aircraft Wake Vortex Separation on Air Transportation Capacity", $4^{\text {th }}$ FAA/Eurocontrol R\&D Conference, Santa Fe, new Mexico, USA

Donahue, G. L., Shorttle, J., Jeddi, B., (2008), "Looking for the Capacity in NGATS", on Transportation Research Board (TRB) Annual Meeting, January 14, Washington DC, USA

EEC, (2005), Optimal Procedures and Techniques for the Improvement of Approach and Landing - OPTIMA, Sixth Framework Program, FP6-2002-Aero 1502880, Deliverable 1.2, European commission, Brussels, Belgium

FAA, (2004), Summary Results from Long-Term Wake Turbulence Measurement at San Francisco International Airport, U.S. Department of Transportation, Federal Aviation Administration, DOT-VNTSC-FA27-PM-04-13, Washington, DC, USA

FAA, (2004a), Airport Capacity Benchmark Report, Federal Aviation Administration, U.S. Department of Transportation, The MITRE Corporation, Washington DC, USA,

FAA, (2006), Aeronautical Information Manual: Official Guide to Basic Flight Information and ATC Procedures", Federal Aviation Administration, Washington D. C., USA

Fraport, (2004), EDDF-SOP - Standard Operating Manual- Frankfurt Main Airport, Virtual Frankfurt Airport SOP V9.6, http:/ / www.vacc-sag.org, Accessed April 15, 2004 
Gilbo, E. P., (1993), “Airport Capacity: Representation Estimation, Optimization”, IEEE Transactions on Control System Technology, Vol. 1, No. 3, 1993, pp. 144-153

Hammer, J., (2000), "A Case Study of Paired Approach Procedure to Closely Spaced Parallel Runways", Air Traffic Control Quarterly, Vol. 8, No. 3, 2000, pp. 223-252

Harris, R. M., (1972), Models for Runway Capacity Analysis, The MITRE Corporation Technical Report, MTR-4102, Rev. 2, Langley, Virginia, USA

Hockaday, S. L. M., Kanafani, A., (1974), "Development in Airport Capacity Analysis", Transportation Research, Vol. 8, No. 3, 171-180

ICAO, (1996), Rules of the air and air Traffic Services: Procedures for Air Navigation Services, Doc. 4444 -RAC/501, 13-th Edition, International Civil Aviation Organization. Montreal, Canada

Ignaccolo, M., (2003), "A Simulation Model for Airport Capacity and Delay Analysis", Transportation Planning and Technology, Vol. 26, No. 2, pp. 135-170

Janic, M., Tosic, V., (1982), “Terminal Airspace Capacity Model”, Transportation Research, Vol. 16A, No. 4, pp. 253-260

Janic, M., (2001), Air Transport System Analysis and Modeling: Capacity, Quality of Service and Economics, Transport Series Books Vol. 16, Taylor and Francis, UK

Janic, M., (2006), "Model of Ultimate Capacity of Dual-Dependent Parallel Runways", Transportation Research Record 1951, pp. 76-85

Janic, M, (2008), "A Model of Runway Landing Capacity Based on the ATC Time-Based Separation Rules", Transportation Research Record (TRB), No. 2052 Aviation, pp. 7989

Janic, M., (2008a), “Modelling the Capacity of Closely-Spaced Parallel Runways Using Innovative Approach Procedures, Transportation Research C, Vol. 16, No. 6, pp. 704730

LMI, (2004), “Business Case for NASA Wake Vortex Technology”, Power-Point

Presentation, Logistics Management Institute, Governmental Consulting, Washington D.C., USA

Newell, G. F., (1979), “Airport Capacity and Delays”, Transportation Science, Vol. 13, No. 3, pp. 201-241

NASA, (1998), Air Traffic and Operational Data on Selected U.S. Airports With Parallel Runways, National Aeronautic and Space Administration, NASA/CR-1998-207675, Langley, Virginia, USA

NASA, (1999), Benefit Estimates of Terminal Area Productivity Program Technologies, NASA/CR - 1999 208989, National Aeronautics and Space Administration, Virginia, USA

NASA, (2001), Enhancement Airport Capacity through Safe Dynamic Reduction of Aircraft Separation: NASA's Aircraft Vortex Spacing System (AVOSS), National Aeronautical and Space Administration, Hampton, Virginia. USA.

ONERA/DOTA, (2005), WV Separation Technology Case: Preparation of Wake Detection Technology Case, EUROCONTROL EEC TRSC42/2004, Public Summary Report Version 1.1, Palaiseau, Cedex, France

Odoni, A. R., Bowman, J., (1997), Existing and Required Modelling Capabilities for Evaluating ATM Systems and Concepts, Final Report No. NAG2-997, International Centre for Air Transportation, Massachusetts Institute of Technology, Massachusetts, Boston, USA

Richetta, O., Odoni, A. R. (1993), "Solving Optimally the Static Ground Holding Policy Problem in Air Traffic Control", Transportation Science, Vol. 27, No. 3, 1993, pp. 228-238 
Richetta, O., Odoni, A. R., (1994), “Dynamic Solution to the Ground -holding Problem in air Traffic Control", Transportation Research A, Vol. 28A, No. 3, pp. 167-185

Richetta, O., (1995), “Optimal Algorithms and a Remarkable Efficient Heuristic for the Ground Holding Problem in Air Traffic Control”, Operations Research, Vol. 43, No. 5, pp. 758770

Robinson III, J. E., Davis, T. J., Isaacson, D. R., (1997), “Fuzzy Reasoning Based Sequencing of Arrival Aircraft in the Terminal Area", The American Institute of Aeronautics and Astronautics (AIAA) Guidance, Navigation, and Control Conference, New Orleans, USA, p. 11, 1997

Sarpkaya, T, (2000), "New Model for Vortex Decay in the Atmosphere", Journal of Aircraft, Vol. 37, No. 1, pp. 53-61

Shortle, J. F., Jeddi, B. G., (2007), “Probabilistic Analysis of Wake Vortex Hazards for Landing Aircraft Using Multilateration Data, 86 th TRB Conference, Transportation Research Board, Washington D.C., USA

Swedish, W. J/. (1981), Upgraded Airfield Capacity Model, The MITRE Corporation Technical Report, MTR-81W16, Vols 1 and 2, Langley, Virginia, USA

TC, (2004), Approval of Steep Approach Landing Capability of Transport Category Aircraft, Aircraft Certification, Civil Aviation, Transport Canada

Terrab, M., Odoni, A. R. (1993), "Strategic Flow Management in Air Traffic Control", Operations Research, Vol. 41, No. 1, pp. 138-152

Winckelmans, G., Desenfans, D., (2006), "Probabilistic Prediction of Wake Position and Strength", Specialist's Report - Final Part II, Research Needs, WakeNet2, Europe

Wu Cheng-Lung, Caves R., (2002), “Research Review of Air Traffic Management." Transport Reviews, Vol. 22, No. 1, pp. 115-132 


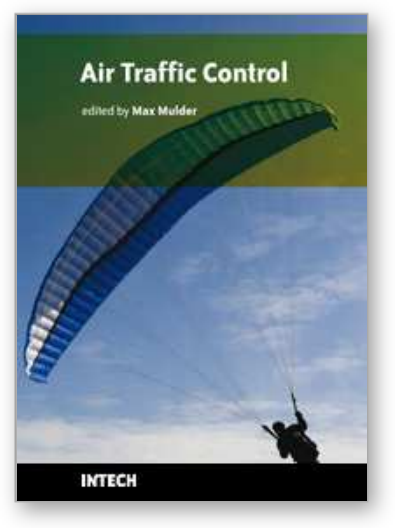

\author{
Air Traffic Control \\ Edited by Max Mulder
}

ISBN 978-953-307-103-9

Hard cover, 172 pages

Publisher Sciyo

Published online 17, August, 2010

Published in print edition August, 2010

Improving air traffic control and air traffic management is currently one of the top priorities of the global research and development agenda. Massive, multi-billion euro programs like SESAR (Single European Sky ATM Research) in Europe and NextGen (Next Generation Air Transportation System) in the United States are on their way to create an air transportation system that meets the demands of the future. Air traffic control is a multi-disciplinary field that attracts the attention of many researchers, ranging from pure mathematicians to human factors specialists, and even in the legal and financial domains the optimization and control of air transport is extensively studied. This book, by no means intended to be a basic, formal introduction to the field, for which other textbooks are available, includes nine chapters that demonstrate the multi-disciplinary character of the air traffic control domain.

\title{
How to reference
}

In order to correctly reference this scholarly work, feel free to copy and paste the following:

Milan Janic (2010). The Potential of Some Innovative Operational Procedures for Increasing of the Airport Runway Capacity, Air Traffic Control, Max Mulder (Ed.), ISBN: 978-953-307-103-9, InTech, Available from: http://www.intechopen.com/books/air-traffic-control/the-potential-of-some-innovative-operational-proceduresfor-increasing-of-the-airport-runway-capacit

\section{INTECH}

open science | open minds

\author{
InTech Europe \\ University Campus STeP Ri \\ Slavka Krautzeka 83/A \\ 51000 Rijeka, Croatia \\ Phone: +385 (51) 770447 \\ Fax: +385 (51) 686166 \\ www.intechopen.com
}

\author{
InTech China \\ Unit 405, Office Block, Hotel Equatorial Shanghai \\ No.65, Yan An Road (West), Shanghai, 200040, China \\ 中国上海市延安西路65号上海国际贵都大饭店办公楼 405 单元 \\ Phone: +86-21-62489820 \\ Fax: +86-21-62489821
}


(C) 2010 The Author(s). Licensee IntechOpen. This chapter is distributed under the terms of the Creative Commons Attribution-NonCommercialShareAlike-3.0 License, which permits use, distribution and reproduction for non-commercial purposes, provided the original is properly cited and derivative works building on this content are distributed under the same license. 